\title{
A Recursive Hypergraph Bipartitioning Framework for Reducing Bandwidth and Latency Costs Simultaneously
}

\author{
Oguz Selvitopi, Seher Acer, and Cevdet Aykanat
}

\begin{abstract}
Intelligent partitioning models are commonly used for efficient parallelization of irregular applications on distributed systems. These models usually aim to minimize a single communication cost metric, which is either related to communication volume or message count. However, both volume- and message-related metrics should be taken into account during partitioning for a more efficient parallelization. There are only a few works that consider both of them and they usually address each in separate phases of a two-phase approach. In this work, we propose a recursive hypergraph bipartitioning framework that reduces the total volume and total message count in a single phase. In this framework, the standard hypergraph models, nets of which already capture the bandwidth cost, are augmented with message nets. The message nets encode the message count so that minimizing conventional cutsize captures the minimization of bandwidth and latency costs together. Our model provides a more accurate representation of the overall communication cost by incorporating both the bandwidth and the latency components into the partitioning objective. The use of the widely-adopted successful recursive bipartitioning framework provides the flexibility of using any existing hypergraph partitioner. The experiments on instances from different domains show that our model on the average achieves up to 52 percent reduction in total message count and hence results in 29 percent reduction in parallel running time compared to the model that considers only the total volume.
\end{abstract}

Index Terms-Communication cost, bandwidth, latency, partitioning, hypergraph, recursive bipartitioning, load balancing, sparse matrix vector multiplication, combinatorial scientific computing

\section{INTRODUCTION}

$F^{-1 n}$ OR irregular applications in the scientific computing domain and several other domains, the intelligent partitioning methods are commonly employed to reduce the communication overhead for efficient parallelization in a distributed setting. Graph and hypergraph partitioning models are ubiquitously utilized in this regard.

\subsection{Motivation and Related Work}

A common cost model for representing the communication requirements of parallel applications consists of the bandwidth and latency components. The bandwidth component is proportional to the amount (volume) of data transferred and the latency component is proportional to the number of messages communicated. In order to capture the communication requirements of parallel applications more accurately, both components should be taken into account in the partitioning models.

Although graph/hypergraph partitioning models that address the bandwidth component are abundant in the literature [1], [2], [3], [4], [5], [6], [7], [8], [9], [10], [11], there exist only a few works that also address the latency component. A relatively early work by Uçar and Aykanat [12]

- The authors are with the Department of Computer Engineering, Bilkent University, Ankara 06800, Turkey.

E-mail: \{reha, acer, aykanat\}@cs.bilkent.edu.tr.

Manuscript received 30 Nov. 2015; revised 18 May 2016; accepted 26 May 2016. Date of publication 6 June 2016; date of current version 18 Jan. 2017.

Recommended for acceptance by D. Trystram.

For information on obtaining reprints of this article, please send e-mail to: reprints@ieee.org, and reference the Digital Object Identifier below.

Digital Object Identifier no. 10.1109/TPDS.2016.2577024 proposes a two-phase approach in which the bandwidth and latency components are respectively addressed in the first and second phases by reducing total communication volume in the former and total message count in the latter. They propose the communication hypergraph model for the second phase to capture the messages and the processors involved. Their method is used for partitioning sparse matrices in the context of iterative solvers for nonsymmetric linear systems and exploits the flexibility of using nonconformal partitions for the vectors in the solver. A recent study by Deveci et al. [13] addresses multiple communication cost metrics via hypergraph partitioning in a single phase. These metrics involve the bandwidth-related metrics such as total volume, maximum send/receive volume, etc. as well as the latency-related metrics such as total message count and maximum send message count. All metrics are addressed in the refinement stage of the partitioning. Their approach introduces an additional cost of $O\left(V K^{2}\right)$ to each refinement pass for handling multiple metrics, where $V$ and $K$ denote the number of tasks in the application and the number of processors, respectively. Another work that is reported to reduce the latency cost in an indirect manner uses the $\lambda(\lambda-1)$ metric in order to correctly encapsulate the total communication volume in the target application [14].

There are studies that address the latency overhead via providing an upper bound on the number of messages communicated [8], [15], [16], [17], [18], [19], [20], [22], [23], [24], [25]. These works usually assume that $K$ processors are organized as a $\sqrt{K} \times \sqrt{K}$ mesh and restrict the communication along the rows and the columns of the processor mesh, which results in $O(\sqrt{K})$ messages for each processor. Most 
of the works bounding the latency component do not explicitly reduce the bandwidth component. The target applications in these works are usually centered around parallelizing sparse matrix computations.

There are a few studies that also aim at reducing volume besides bounding the message count. Çatalyürek and Aykanat [8] propose a two-phase method that makes use of hypergraph partitioning to achieve a Cartesian distribution of sparse matrices, namely 2D checkerboard partitioning. In the first phase, they obtain a rowwise $\sqrt{K}$-way partition and in the second phase, they use multiple vertex weights determined from the partition information of the first phase and obtain a columnwise $\sqrt{K}$-way partition. In both phases, the objective is to minimize the total volume. Boman et al. [23] achieves a similar feat with a faster method for scale-free graphs, again in two phases. In the first phase, their approach can make use of any available graph/hypergraph partitioner to obtain a $1 \mathrm{D}$ vertex partition. In the second phase, they use an effective algorithm to redistribute the nonzeros in the off-diagonal blocks to guarantee the $O(\sqrt{K})$ upper bound. These two methods are proposed for efficient parallelization of sparse matrix vector multiplication.

\subsection{Contributions}

Most of the existing graph/hypergraph partitioning models in the literature address only the bandwidth component while ignoring the latency component. In this work, we propose an augmentation to the existing models in order to minimize the bandwidth and the latency components simultaneously in a single phase. Our approach relies on the commonly adopted recursive bipartitioning (RB) framework [1], [5], [26], [27], [28]. The RB framework recursively partitions a given domain of computational tasks and data items into two until desired number subdomains is obtained. Consider a subdomain to be bipartitioned and the set of data items in this subdomain that are required by the tasks in some other subdomain. Keeping these items together in the bipartitioning ensures only one of the new subdomains to send a message to that other subdomain, avoiding an increase in the total number of messages. In order to encourage keeping these items together, we introduce message nets to the standard hypergraph model so that dividing these items is penalized with a cost equal to startup latency. The nets of the standard hypergraph model are referred to as the volume nets and with the addition of the message nets, this augmented hypergraph now contains both the volume and message nets. Partitioning this hypergraph presents a more accurate picture of the communication cost model as the objective of minimizing the cutsize in the partitioning encapsulates the reduction of both the total volume and the total message count.

Our approach is tailored for the parallel applications in which there exists a single communication phase, that is either preceded or succeeded by a computational phase. The parallel application is also assumed to be performed iteratively and a conformal partition on input and output data is required, where the input of the next iteration is obtained from the output of the current iteration. These common assumptions are suited well to the needs of several applications from various domains. Compared to the standard hypergraph partitioning model in which only the bandwidth component is minimized [1], our approach introduces an additional cost of $O\left(p \log _{2} K\right)$ due to the addition of the message nets, where $p$ is the number of pins in the hypergraph. The proposed model does not depend on a specific hypergraph partitioning tool implementation, hence it can make use of any hypergraph partitioner such as $\mathrm{PaToH}$ [1], [29], hMetis [26] or Mondriaan [7]. In our experiments, we consider 1D parallel sparse matrix vector multiplication (SpMV) as an example application. Our approach is shown to be effective at reducing the latency component as it attains an 18-52 percent reduction in the total number of messages at the expense of an 8-70 percent increase in the total volume compared to the standard model. The experiments validate the necessity of addressing both communication components as the proposed model reduces the parallel running time of SpMV up to 29 percent for 2048 processors on the average.

The rest of the paper is organized as follows. Section 2 describes the properties of the target applications and how to model them with hypergraphs for parallelization. The proposed hypergraph partitioning model and its extensions are given in Section 3. Section 4 evaluates the proposed model in terms of both the communication statistics and the parallel running time of SpMV. Section 5 concludes.

\section{BACKGROUND}

\subsection{Hypergraph Partitioning Problem}

A hypergraph $\mathcal{H}=(\mathcal{V}, \mathcal{N})$ is defined as a set of $n$ vertices $\mathcal{V}=\left\{v_{1}, \ldots, v_{n}\right\}$ and a set of $m$ nets $\mathcal{N}=\left\{n_{1}, \ldots, n_{m}\right\}$. Each net $n_{j} \in \mathcal{N}$ connects a subset of vertices, which is denoted by $\operatorname{Pins}\left(n_{j}\right) \subseteq \mathcal{V}$. The set of nets that connect $v_{i}$ is denoted by $\operatorname{Nets}\left(v_{i}\right) \subseteq \mathcal{N}$. Each vertex $v_{i}$ has an associated weight $w\left(v_{i}\right)$ and each net $n_{j}$ has an associated cost $c\left(n_{j}\right)$.

$\Pi=\left\{\mathcal{V}_{1}, \ldots, \mathcal{V}_{K}\right\}$ is said to be a $K$-way vertex partition of $\mathcal{H}$ if parts are mutually disjoint and exhaustive. In $\Pi$, net $n_{j}$ is said to connect part $\mathcal{V}_{k}$ if it connects at least one vertex in $\mathcal{V}_{k}$, i.e., $\operatorname{Pins}\left(n_{j}\right) \cap \mathcal{V}_{k} \neq \emptyset$. The connectivity set $\Lambda\left(n_{j}\right)$ of $n_{j}$ is defined as the set of parts connected by $n_{j}$. The connectivity of $n_{j}, \lambda\left(n_{j}\right)$, denotes the number of parts connected by $n_{j} . n_{j}$ is said to be cut if it connects more than one part, i.e., $\lambda\left(n_{j}\right)>1$, and uncut otherwise.

The hypergraph partitioning problem is defined as finding a $K$-way vertex partition $\Pi$ with the objective of minimizing cutsize, which is defined as

$$
\operatorname{cut}(\Pi)=\sum_{n_{j} \in \mathcal{N}^{\text {cut }}} c\left(n_{j}\right)\left(\lambda\left(n_{j}\right)-1\right),
$$

subject to the balance constraint

$$
W\left(\mathcal{V}_{k}\right) \leq(1+\epsilon) W_{\text {avg }},
$$

where $\mathcal{N}^{\text {cut }}$ denotes the set of cut nets, $W\left(\mathcal{V}_{k}\right)=\sum_{v_{i} \in \mathcal{V}_{k}} w\left(v_{i}\right)$ denotes the weight of $\mathcal{V}_{k}, W_{\text {avg }}=\sum_{k} W\left(\mathcal{V}_{k}\right) / K$ denotes the average part weight and $\epsilon$ denotes the maximum allowed imbalance ratio. The hypergraph partitioning problem is NP-hard [30].

\subsection{Recursive Hypergraph Bipartitioning}

Our work relies on recursive bipartitioning, hence we give the relevant notation. In $\mathrm{RB}$, a given hypergraph $\mathcal{H}$ is 


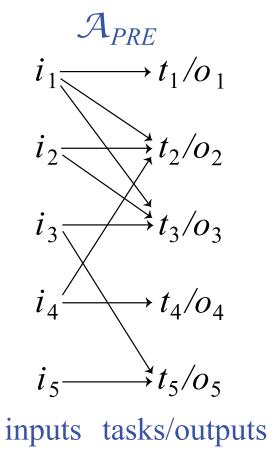

(a)

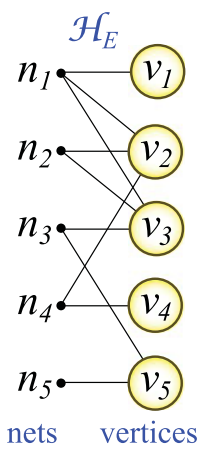

(b)

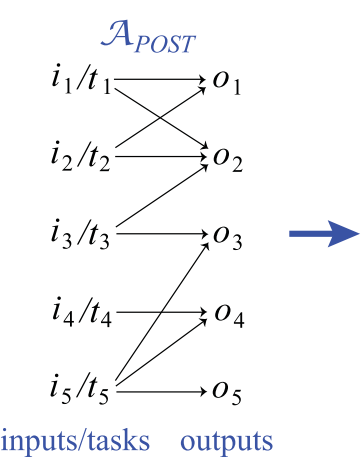

(a)



(b)
Fig. 1. (a) An example for $A_{P R E}$. (b) The hypergraph $\mathcal{H}_{E}$ that represents $A_{P R E}$.

recursively partitioned into two parts until $K$ parts are obtained. Obtaining a $K$-way partition of $\mathcal{H}$ through RB induces a binary tree with $\left\lceil\log _{2} K\right\rceil$ levels, which is referred to as an RB tree. For the sake of simplicity, we assume $K$ is a power of two. The $\ell$ th level of the RB tree contains $2^{\ell}$ hypergraphs, denoted with $\mathcal{H}_{0}^{\ell}, \ldots, \mathcal{H}_{2^{\ell}-1}^{\ell}$ from left to right, $0 \leq \ell \leq \log _{2} K$. A bipartition $\Pi=\left\{\mathcal{V}_{L}, \mathcal{V}_{R}\right\}$ on hypergraph $\mathcal{H}_{k}^{\ell}$ in the $\ell$ th level forms two new vertex-induced hypergraphs $\mathcal{H}_{2 k}^{\ell+1}=\left(\mathcal{V}_{L}, \mathcal{N}_{L}\right)$ and $\mathcal{H}_{2 k+1}^{\ell+1}=\left(\mathcal{V}_{R}, \mathcal{N}_{R}\right)$, both in level $\ell+1$. Here, $\mathcal{V}_{L}$ and $\mathcal{V}_{R}$ are respectively used to refer to the left and right part of the bipartition without loss of generality. A single bipartitioning is also referred to as an RB step.

The net sets of the newly formed hypergraphs in an RB step are constructed via cut-net splitting method [1] in order to capture the cutsize (1). In this method, a cut-net $n_{j}$ in $\Pi=\left\{\mathcal{V}_{L}, \mathcal{V}_{R}\right\}$ is split into two new nets $n_{j}^{L} \in \mathcal{N}_{L}$ and $n_{j}^{R} \in \mathcal{N}_{R}$, where $\operatorname{Pins}\left(n_{j}^{L}\right)=\operatorname{Pins}\left(n_{j}\right) \cap \mathcal{V}_{L}$ and $\operatorname{Pins}\left(n_{j}^{R}\right)=$ $\operatorname{Pins}\left(n_{j}\right) \cap \mathcal{V}_{R}$. Internal nets of $\mathcal{V}_{L}$ and $\mathcal{V}_{R}$ are respectively included in $\mathcal{N}_{L}$ and $\mathcal{N}_{R}$.

\subsection{Parallelizing Applications}

\subsubsection{Target Application Properties}

Consider an application $\mathcal{A}=(\mathcal{I}, \mathcal{T}, \mathcal{O})$ to be parallelized, where $\mathcal{I}=\left\{i_{1}, \ldots, i_{I}\right\}$ is the set of input data items, $\mathcal{T}=\left\{t_{1}, t_{T}\right\}$ is the set of tasks and $\mathcal{O}=\left\{o_{1}, \ldots, o_{O}\right\}$ is the set of output data items. $I, T$ and $O$ respectively denote the sizes of $\mathcal{I}, \mathcal{T}$ and $\mathcal{O}$. The items and tasks of this application constitute a domain to be partitioned for parallelization. The tasks operate on input items and produce output items. There is no dependency among tasks, however there is interaction among the tasks that need the same input as well as the tasks that contribute to the same output. Input $i_{j} \in \mathcal{I}$ is required by a subset of tasks, denoted by $\operatorname{tasks}\left(i_{j}\right) \subseteq \mathcal{T}$. A subset of tasks produce intermediate results for output $o_{j} \in \mathcal{O}$, again denoted by $\operatorname{tasks}\left(o_{j}\right) \subseteq \mathcal{T}$. size $\left(t_{i}\right)$ denotes the amount of time required to complete task $t_{i}$ and size $\left(i_{j}\right)\left(\operatorname{size}\left(o_{j}\right)\right)$ denotes the storage size of item $i_{j}\left(o_{j}\right)$. The tasks are atomic, i.e., each task is processed exactly by one processor. In a parallel setting, tasks and items are distributed among a number of processors.

We focus on applications in which either the intermediate results for $o_{j}$ are produced by a single task for each $o_{j} \in \mathcal{O}\left(\left|\operatorname{tasks}\left(o_{j}\right)\right|=1\right)$ or $i_{j}$ is required by a single task for each $i_{j} \in \mathcal{I}\left(\left|\operatorname{tasks}\left(i_{j}\right)\right|=1\right)$. In a distributed setting, there is
Fig. 2. (a) An example for $A_{P O S T}$. (b) The hypergraph $\mathcal{H}_{F}$ that represents $A_{P O S T}$.

only a single communication phase in both cases, in which either only the inputs or only the intermediate results of the outputs are communicated. The applications that exhibit the properties in the former and the latter cases are respectively denoted with $A_{P R E}$ and $A_{P O S T}$. In $A_{P R E}$, the communications are performed in a so-called pre-communication phase, whereas in $A_{P O S T}$, the communications are performed in a so-called post-communication phase.

In $A_{P R E}$, the processor responsible for task $t_{j}$ is also held responsible for storing output $o_{j}$. First, for each input $i_{k}$, the processor that stores $i_{k}$ sends it to each processor which is responsible for at least one task in $\operatorname{tasks}\left(i_{k}\right)$. This communication operation on $i_{k}$ is referred to as an expand operation. Then, the processor responsible for $t_{j}$ executes it by operating on inputs $\left\{i_{k}: t_{j} \in \operatorname{tasks}\left(i_{k}\right)\right\}$ to compute the result for $o_{j}$ in a communication-free manner. An example for $A_{P R E}$ is illustrated in Fig. 1a.

In $A_{P O S T}$, the processor responsible for task $t_{j}$ is also held responsible for storing input $i_{j}$. First, the processor responsible for $t_{j}$ executes it on $i_{j}$ in a communication-free manner and produces intermediate results for the outputs $\left\{o_{k}: t_{j} \in \operatorname{tasks}\left(o_{k}\right)\right\}$. Then, the processor responsible for $o_{k}$ receives corresponding intermediate results from each processor which is responsible for at least one task in tasks $\left(o_{k}\right)$ and reduces them through an associative and/or commutative operator. This communication operation on $o_{k}$ is referred to as a fold operation. An example for $A_{P O S T}$ is illustrated in Fig. 2a.

We assume that $A_{P R E}$ and $A_{P O S T}$ accommodate the following common properties: (i) they are performed repeatedly, (ii) the number of input and output items are equal, and (iii) there exists a one-to-one dependency between input and output items through successive iterations, i.e., output $o_{j}$ of the current iteration is used to obtain input $i_{j}$ of the next iteration. Note that if this one-to-one dependency is not respected in assigning items to processors, redundant communication is incurred. For this reason, a conformal partition on input and output items should be adopted in which $i_{j}$ and $o_{j}$ are assigned to the same processor.

\subsubsection{Hypergraph Models for $A_{P R E}$ and $A_{P O S T}$}

We use hypergraphs $\mathcal{H}_{E}=\left(\mathcal{V}_{E}, \mathcal{N}_{E}\right)$ and $\mathcal{H}_{F}=\left(\mathcal{V}_{F}, \mathcal{N}_{F}\right)$ to represent $A_{P R E}$ and $A_{P O S T}$, respectively. Subscripts "E" and "F" are used to denote the fact that $A_{P R E}$ and $A_{P O S T}$ contain "Expand" and "Fold" operations, respectively. In both $\mathcal{H}_{E}$ and $\mathcal{H}_{F}$, the vertices represent tasks and items, i.e., $\mathcal{V}_{E}=\mathcal{V}_{F}=\left\{v_{1}, \ldots, v_{T}\right\}$, where $v_{i}$ represents task $t_{i}$ together 




(a)



(b)

Fig. 3. Two 3-way partitionings of the same hypergraph $A_{P R E}$. Only the parts of $v_{3}, v_{6}$ and $v_{7}$ differ in $\Pi_{A}$ and $\Pi_{B}$. $\Pi_{A}$ incurs less volume but more messages while $\Pi_{B}$ incurs more volume but less messages. Note that $v_{j}$ represents both task $t_{j}$ and input $i_{j}$.

with possibly multiple input-output pairs $\left(i_{j}, o_{j}\right)$ such that $\operatorname{tasks}\left(o_{j}\right)=\left\{t_{i}\right\}$ for $A_{P R E}$ and $\operatorname{tasks}\left(i_{j}\right)=\left\{t_{i}\right\}$ for $A_{P O S T}$. The weight of a vertex $w\left(v_{i}\right)$ in both $\mathcal{H}_{E}$ and $\mathcal{H}_{F}$ is assigned the amount of time required to execute $t_{i}$, i.e., $w\left(v_{i}\right)=\operatorname{size}\left(t_{i}\right)$. Both net sets $\mathcal{N}_{E}$ and $\mathcal{N}_{F}$ consist of $I=O$ nets, $\mathcal{N}_{E}=\mathcal{N}_{F}=\left\{n_{1}, \ldots, n_{I=O}\right\}$. The nets in $\mathcal{N}_{E}$ capture the interactions between tasks and inputs: For each input $i_{j}$, there exists an expand net $n_{j}$ to represent the expand operation on $i_{j}$ with $\operatorname{Pins}\left(n_{j}\right)=\left\{v_{i}: t_{i} \in \operatorname{tasks}\left(i_{j}\right)\right\}$. The nets in $\mathcal{N}_{F}$ capture the interactions between tasks and outputs: For each output $o_{j}$, there exists a fold net $n_{j}$ to represent the fold operation on $o_{j}$ with $\operatorname{Pins}\left(n_{j}\right)=\left\{v_{i}: t_{i} \in \operatorname{tasks}\left(o_{j}\right)\right\}$. The cost of an expand net $n_{j} \in \mathcal{N}_{E}$ is assigned the size of the respective input $i_{j}$ multiplied with $t_{w}$, i.e., $c\left(n_{j}\right)=\operatorname{size}\left(i_{j}\right) t_{w}$. In a similar manner, the cost of a fold net $n_{j} \in \mathcal{N}_{F}$ is assigned the size of the respective output $o_{j}$ multiplied with $t_{w}$, i.e., $c\left(n_{j}\right)=\operatorname{size}\left(o_{j}\right) t_{w}$. Here, $t_{w}$ is the time required to transfer a single unit of data item. Figs. $1 \mathrm{~b}$ and $2 \mathrm{~b}$ display the hypergraphs $\mathcal{H}_{E}$ and $\mathcal{H}_{F}$ that respectively represent the example applications in Figs. 1 a and 2 a.

A $K$-way partition of $\mathcal{H}_{E} / \mathcal{H}_{F}$ is decoded to obtain a distribution of tasks and data items among $K$ processors. The responsibility of executing tasks and storing items in the subdomain represented by part $\mathcal{V}_{k}$ is, without loss of generality, assigned to processor $P_{k}$. A cut-net $n_{j}$ in the partition of $\mathcal{H}_{E}$ necessitates an expand operation on input $i_{j}$ from the processor that stores $i_{j}$ to $\lambda\left(n_{j}\right)-1$ processors, whereas a cut-net $n_{j}$ in the partition of $\mathcal{H}_{F}$ necessitates a fold operation on the intermediate results for output $o_{j}$ from $\lambda\left(n_{j}\right)-1$ processors to the processor that stores $o_{j}$. These operations respectively amount to $\operatorname{size}\left(i_{j}\right)\left(\lambda\left(n_{j}\right)-1\right)$ and $\operatorname{size}\left(o_{j}\right)$ $\left(\lambda\left(n_{j}\right)-1\right)$ volume of communication units. The partitioning constraint of maintaining balance on part weights (2) in both $\mathcal{H}_{E}$ and $\mathcal{H}_{F}$ corresponds to maintaining balance on the processors' expected execution time. The partitioning objective of minimizing cutsize (1) in both $\mathcal{H}_{E}$ and $\mathcal{H}_{F}$ corresponds to minimizing the total communication volume incurred in pre-/post-communication phases.

\subsubsection{Examples for $A_{P R E}$ and $A_{P O S T}$}

We consider parallel sparse matrix vector multiplication (SpMV) $y \leftarrow A x$ performed in a repeated manner (such as in iterative solvers) which is a common kernel in scientific computing. Here, $A$ is an $n \times n$ matrix, and $x$ and $y$ are vectors of size $n$. In SpMV, the inputs are $x$-vector elements, i.e., $\mathcal{I}=\left\{x_{1}, \ldots, x_{n}\right\}$, and the outputs are $y$-vector elements, i.e., $\mathcal{O}=\left\{y_{1}, \ldots, y_{n}\right\}$, where $x_{j}$ and $y_{i}$ respectively denote the $j$ th $x$-vector element and $i$ th $y$-vector element. A conformal partition on input and output vectors is usually preferred in order to avoid redundant communication.

1D row-parallel SpMV and 1D column-parallel SpMV are examples for $A_{P R E}$ and $A_{P O S T}$. In 1D row-parallel SpMV, task $t_{j}$ stands for the inner product $\left\langle a_{j *}, x\right\rangle$, while in 1D column-parallel SpMV, $t_{j}$ stands for the scalar multiplication $x_{j} a_{* j}$, where $a_{j *}$ and $a_{* j}$ respectively denote the $j$ th row and $j$ th column of $A$, for $1 \leq j \leq n$. The size of $t_{j}$ is equal to the number of nonzeros in $j$ th row and $j$ th column of $A$, respectively in $A_{P R E}$ and $A_{P O S T}$, i.e., the number of multiply-andadd operations in $\left\langle a_{j *}, x\right\rangle$ and $x_{j} a_{* j}$. In both, there exist a total of $n$ inputs, $n$ tasks and $n$ outputs. In 1D row-parallel SpMV, input $x_{j}$ is required by each inner product $\left\langle a_{i *}, x\right\rangle$ such that $a_{i *}$ contains a nonzero in $j$ th column, that is, $\operatorname{tasks}\left(x_{j}\right)=\left\{t_{i}: a_{i j} \neq 0\right\}$. The intermediate results for each output $y_{j}$ are produced only by the task $\left\langle a_{j *}, x\right\rangle$, i.e., $\operatorname{tasks}\left(y_{j}\right)=\left\{t_{j}\right\}$. In 1D column-parallel SpMV, input $x_{j}$ is required only by the task $x_{j} a_{* j}$, i.e., $\operatorname{tasks}\left(x_{j}\right)=\left\{t_{j}\right\}$. Each task $x_{i} a_{* i}$ produces an intermediate result for output $y_{j}$ such that $a_{* i}$ contains a nonzero in its $j$ th row, that is, $\operatorname{tasks}\left(y_{j}\right)=\left\{t_{i}: a_{j i} \neq 0\right\}$. We represent $1 \mathrm{D}$ row-parallel and 1D column-parallel SpMVs respectively with $\mathcal{H}_{E}=$ $\left(\mathcal{V}_{E}, \mathcal{N}_{E}\right)$ and $\mathcal{H}_{F}=\left(\mathcal{V}_{F}, \mathcal{N}_{F}\right)$. The cost of net $n_{j}$ in both $\mathcal{H}_{E}$ and $\mathcal{H}_{F}$ is assigned $t_{w}$ since it incurs the communication of a single item if it is cut. $\mathcal{H}_{E}$ and $\mathcal{H}_{F}$ are respectively called the column-net and row-net hypergraph models and they are proposed in [1].

The objective of partitioning $\mathcal{H}_{E} / \mathcal{H}_{F}$ correctly captures the total volume while disregarding the message count (Section 2.3.2). To illustrate the aspects of different partitions on these two metrics, we present a motivating example in Fig. 3, where the same $\mathcal{H}_{E}$ is three-way partitioned in two different ways. The arrows represent the messages between processors that are associated with the parts in the figure. For example, in Fig. $3 \mathrm{a}, i_{2}\left(=v_{2}\right)$ needs to be sent from $\mathcal{P}_{1}$ to $\mathcal{P}_{2}$ since it is stored by $\mathcal{P}_{1}$ and the tasks $t_{4}\left(=v_{4}\right)$ and $t_{5}\left(=v_{5}\right)$ 
in $\mathcal{P}_{2}$ need it ( $n_{2}$ captures this dependency). The contents of the messages are indicated next to the arrows. In the first partition $\Pi_{A}$ in Fig. 3a, there are five messages and six communicated items, making up a total of $5 t_{s}+6 t_{w}$ communication cost. In the second partition $\Pi_{B}$ in Fig. $3 \mathrm{~b}$, there are three messages and seven communicated items, making up a total of $3 t_{s}+7 t_{w}$ communication cost. Partitioning $\mathcal{H}_{E}$ is more likely to produce $\Pi_{A}$ since total volume is lower in $\Pi_{A}$ as nets of $\mathcal{H}_{E}$ encode volume. However, $\Pi_{B}$ is more desirable since $3 t_{s}+7 t_{w}$ is less than $5 t_{s}+6 t_{w}$ as $t_{s}$ is usually much larger than $t_{w}$.

\section{Simultaneous Reduction of BandWidth AND LATENCY COSTS}

We consider parallelizations of $A_{P R E}$ and $A_{P O S T}$ via $K$-way partitions on $\mathcal{H}_{E}$ and $\mathcal{H}_{F}$. We describe our model first for $A_{P R E}$ in detail (Section 3.1) and then show how to apply it to $A_{P O S T}$ (Section 3.2.1), as they are dual of each other. Hereinafter, we refer to $\mathcal{H}_{E}$ as $\mathcal{H}$ and $\Pi_{E}$ as $\Pi$. We first assume that $I=O=T$ and describe the model for this case and then extend it to the more general case $I=O \geq T$ (Section 3.2.2).

\subsection{Encoding Messages in Recursive Hypergraph Bipartitioning}

Consider a recursive bipartitioning tree being produced in a breadth-first manner to obtain a $K$-way partition of $\mathcal{H}=(\mathcal{V}, \mathcal{N})$. Let the RB process be currently at the $\ell$ th level, prior to bipartitioning hypergraph $\mathcal{H}_{i}^{\ell}$ in this level. There are currently $2^{\ell}+i$ hypergraphs, enumerated from left to right, $\mathcal{H}_{i}^{\ell}, \ldots, \mathcal{H}_{2 \ell-1}^{\ell}, \mathcal{H}_{0}^{\ell+1}, \ldots, \mathcal{H}_{2 i-1}^{\ell+1}$, at the leaf nodes of the RB tree: $2^{\ell}-i$ of them at level $\ell$ and $2 i$ of them at level $\ell+1$. The vertex sets of these hypergraphs induce a $\left(2^{\ell}+i\right)$-way vertex partition

$$
\Pi_{\text {cur }}(\mathcal{H})=\left\{\mathcal{V}_{i}^{\ell}, \ldots, \mathcal{V}_{2^{\ell}{ }_{-1}}^{\ell}, \mathcal{V}_{0}^{\ell+1}, \ldots, \mathcal{V}_{2 i-1}^{\ell+1}\right\}
$$

This vertex partition is also assumed to induce a $\left(2^{\ell}+i\right)$-way processor partition $\mathbb{P}_{\text {cur }}=\left\{\mathcal{P}_{i}^{\ell}, \ldots, \mathcal{P}_{2^{\ell}-1}^{\ell}, \mathcal{P}_{0}^{\ell+1}, \ldots, \mathcal{P}_{2 i-1}^{\ell+1}\right\}$, where processor group $\mathcal{P}_{i}^{\ell}$ is held responsible for the items / tasks that are in the subdomain represented by $\mathcal{V}_{i}^{\ell}$. An example $\Pi_{\text {cur }}$ is seen in the upper RB tree in Fig. 4.

We refer to the current hypergraph to be bipartitioned $\mathcal{H}_{i}^{\ell}$ as $\mathcal{H}_{\text {cur }}=\left(\mathcal{V}_{\text {cur }}=\mathcal{V}_{i}^{\ell}, \mathcal{N}_{\text {cur }}=\mathcal{N}_{i}^{\ell}\right)$. This bipartitioning generates $\Pi\left(\mathcal{H}_{\text {cur }}\right)=\left\{\mathcal{V}_{L}, \mathcal{V}_{R}\right\}$ and forms two new hypergraphs $\mathcal{H}_{L}=\left(\mathcal{V}_{L}, \mathcal{N}_{L}\right)$ and $\mathcal{H}_{R}=\left(\mathcal{V}_{R}, \mathcal{N}_{R}\right)$. Note that $\mathcal{H}_{L}=\mathcal{H}_{2 i}^{\ell+1}$ and $\mathcal{H}_{R}=\mathcal{H}_{2 i+1}^{\ell+1}$. After bipartitioning, there now exist $2^{\ell}+i+1$ hypergraphs at the leaf nodes and their vertex sets induce a $\left(2^{\ell}+i+1\right)$-way partition:

$$
\Pi_{\text {new }}(\mathcal{H})=\Pi_{\text {cur }}(\mathcal{H})-\left\{\mathcal{V}_{\text {cur }}\right\} \cup\left\{\mathcal{V}_{L}, \mathcal{V}_{R}\right\}
$$

Bipartitioning $\mathcal{V}_{\text {cur }}$ into $\mathcal{V}_{L}$ and $\mathcal{V}_{R}$ is assumed to also bipartition the processor group $\mathcal{P}_{\text {cur }}$ into two processor groups $\mathcal{P}_{L}$ and $\mathcal{P}_{R}$. In accordance, $\mathbb{P}_{\text {new }}=\mathbb{P}_{\text {cur }}-\left\{\mathcal{P}_{\text {cur }}\right\} \cup\left\{\mathcal{P}_{L}, \mathcal{P}_{R}\right\}$.

Fig. 4 displays the two states of the RB tree before and after bipartitioning $\mathcal{H}_{\text {cur }}$ and highlights the messages communicated. Let $M_{\text {cur }}$ be the number of messages between $\mathcal{P}_{\text {cur }}$ and $\mathbb{P}_{\text {cur }}-\left\{\mathcal{P}_{\text {cur }}\right\}$ and $M_{\text {new }} \geq M_{\text {cur }}$ be the number of messages between $\left\{\mathcal{P}_{L}, \mathcal{P}_{R}\right\}$ and $\mathbb{P}_{\text {new }}-\left\{\mathcal{P}_{L}, \mathcal{P}_{R}\right\} . M_{\text {new }}$ can be at
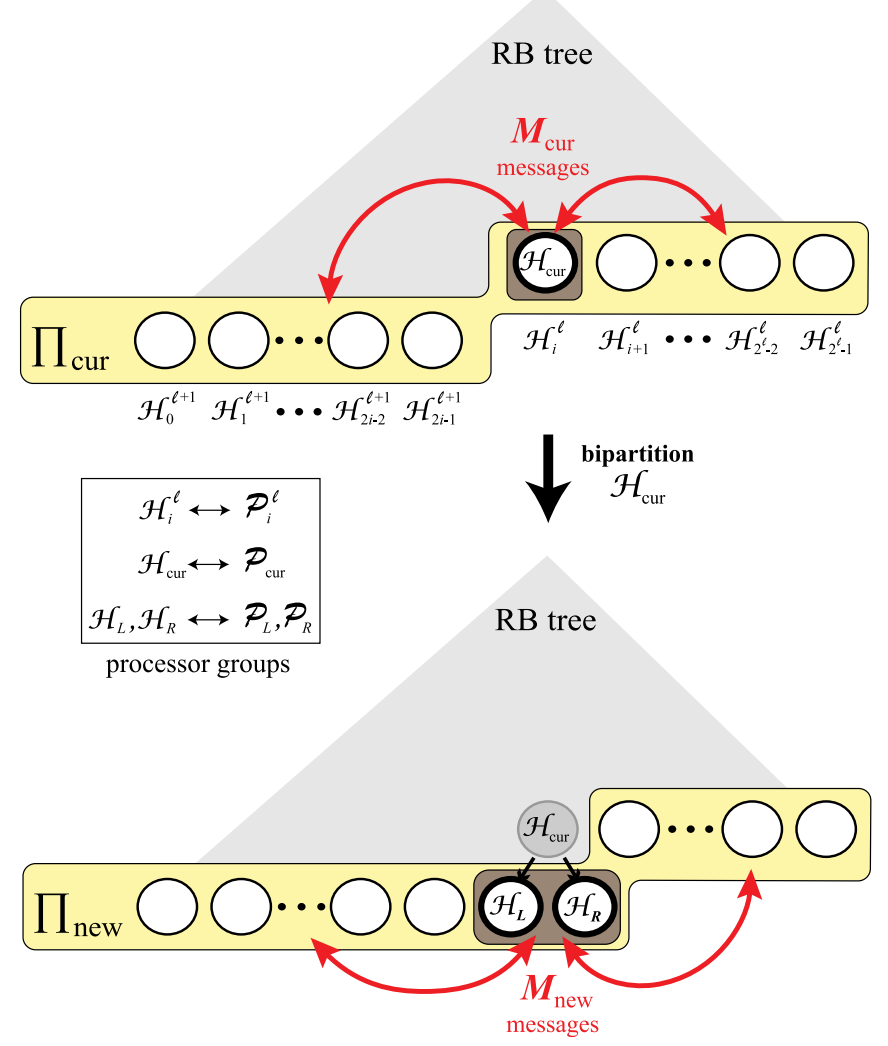

Fig. 4. The state of the RB tree and the number of messages from/to $\mathcal{P}_{\text {cur }}$ and $\left\{\mathcal{P}_{L}, \mathcal{P}_{R}\right\}$ to/from the other processor groups before and after bipartitioning $\mathcal{H}_{\text {cur }}$. The processor groups corresponding to the vertex sets of the hypergraphs are shown in the box.

most $2 M_{\text {cur }}$ which occurs when both $\mathcal{P}_{L}$ and $\mathcal{P}_{R}$ communicate with every $\mathcal{P}_{k}$ that $\mathcal{P}_{\text {cur }}$ communicates with. A new message is incurred when items/tasks that necessitate a message between $\mathcal{P}_{\text {cur }}$ and $\mathcal{P}_{k}$ get scattered across $\mathcal{P}_{L}$ and $\mathcal{P}_{R}$. Consequently, after bipartitioning, both $\mathcal{P}_{L}$ and $\mathcal{P}_{R}$ communicate with $\mathcal{P}_{k}$. Here, the idea is to find a way for items/tasks which as a whole necessitate a message between $\mathcal{P}_{\text {cur }}$ and $\mathcal{P}_{k}$ to be assigned together to either $\mathcal{P}_{L}$ or $\mathcal{P}_{R}$ so that only one of them communicates with $\mathcal{P}_{k}$. By doing so, the goal is to keep the number of messages between $\left\{\mathcal{P}_{L}, \mathcal{P}_{R}\right\}$ and the remaining processor groups in $\mathbb{P}_{\text {new }}$ as small as possible.

To this end, we define new nets, referred to as message nets, to keep the vertices corresponding to items/tasks that necessitate messages altogether. We extend $\mathcal{H}_{\text {cur }}=\left(\mathcal{V}_{\text {cur }}\right.$, $\left.\mathcal{N}_{\text {cur }}\right)$ to $\mathcal{H}_{\text {cur }}^{M}=\left(\mathcal{V}_{\text {cur }}, \mathcal{N}_{\text {cur }}^{M}\right)$ by adding message nets and keeping the expand nets as is, referred to as volume nets. We include both volume and message nets in $\mathcal{H}_{\text {cur }}^{M}$ in order to reduce the total volume and the total message count simultaneously. The following sections define message nets and present an algorithm for forming them.

\subsubsection{Message Nets}

Recall that a vertex $v_{j}$ in $\mathcal{V}$ represents input $i_{j}$ besides task $t_{j}$ and output $o_{j}$, and the processor that stores $i_{j}$ is also held responsible for the possible expand operation on $i_{j}$. Since net $n_{j}$ represents this expand operation, for convenience, we define a function $\operatorname{src}: \mathcal{N} \rightarrow \mathcal{V}$, that maps each original net $n_{j} \in \mathcal{N}$ to its source vertex $\operatorname{src}\left(n_{j}\right) \in \mathcal{V}$, where $\operatorname{src}\left(n_{j}\right)=v_{j}$. 


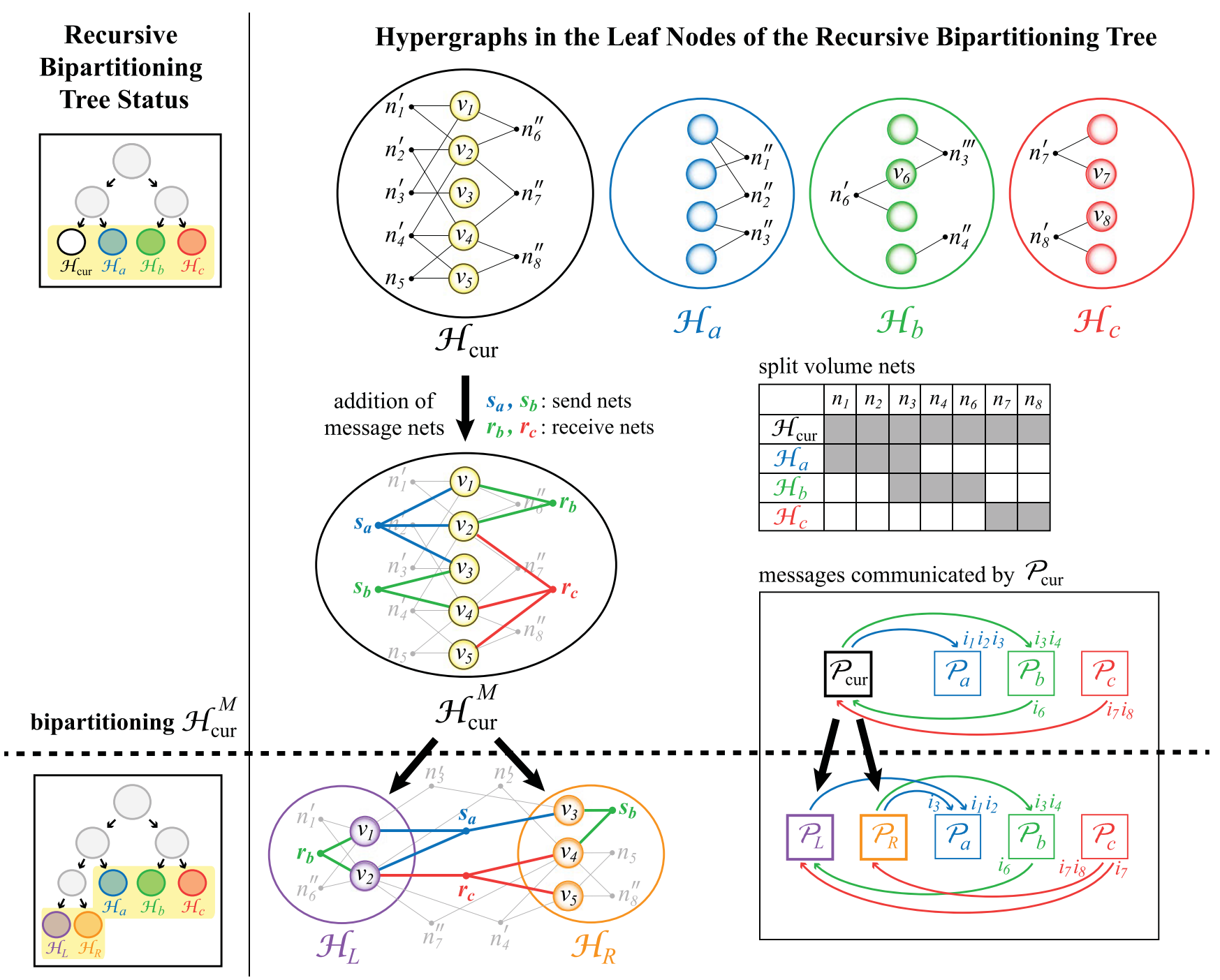

Fig. 5. The illustration of formation and addition of message nets to $\mathcal{H}_{\text {cur }}$ and bipartitioning of $\mathcal{H}_{\text {cur }}^{M}$. Initially, there are four hypergraphs in the leaf nodes of the RB tree: $\mathcal{H}_{c u r}, \mathcal{H}_{a}, \mathcal{H}_{b}$ and $\mathcal{H}_{c}$. Two send $\left(s_{a}\right.$ and $\left.s_{b}\right)$ and two receive $\left(r_{b}\right.$ and $\left.r_{c}\right)$ message nets are added to form $\mathcal{H}_{\text {cur }}^{M}$. Then, $\mathcal{H}_{c u r}^{M}$ is bipartitioned to obtain $\mathcal{H}_{L}$ and $\mathcal{H}_{R}$. The split nets are shown in a table where a shaded entry indicates the existence of the split net in the respective hypergraph. The messages communicated among the respective processor groups are illustrated in the frame at the right bottom corner, where $\mathcal{P}_{\text {cur }}$, $\mathcal{P}_{a}, \mathcal{P}_{b}$ and $\mathcal{P}_{c}$ are respectively associated with $\mathcal{H}_{\text {cur }}, \mathcal{H}_{a}, \mathcal{H}_{b}$ and $\mathcal{H}_{c}$. The colors of the message nets indicate the processor groups that the respective messages are sent to or received from. The dashed line separates the hypergraphs/processor groups before and after bipartitioning. The volume nets in $\mathcal{H}_{\text {cur }}^{M}, \mathcal{H}_{L}$ and $\mathcal{H}_{R}$ are faded out to attract the focus on the message nets.

To aid the discussions in this section, we present an example RB tree in Fig. 5 that currently consists of four leaf hypergraphs $\mathcal{H}_{\text {cur }}, \mathcal{H}_{a}, \mathcal{H}_{b}$ and $\mathcal{H}_{c}$, whose vertex sets form four-way partition $\Pi_{\text {cur }}$. We refer to the nets in given $\mathcal{H}=\mathcal{H}_{0}^{0}$ as original nets and use these nets in describing the algorithm for forming the message nets. An original net may split several times during RB or it may not split by being uncut in the bipartitionings it takes part in. For example in Fig. 5 , the original net $n_{3}$ has split three times, producing $n_{3}^{\prime}$, $n_{3}^{\prime \prime}$ and $n_{3}^{\prime \prime \prime}$ in $\mathcal{H}_{\text {cur }}, \mathcal{H}_{a}$ and $\mathcal{H}_{b}$, respectively. Observe that the vertices connected by $n_{3}$ in $\mathcal{H}$ are equal to the union of the vertices connected by $n_{3}^{\prime}, n_{3}^{\prime \prime}$ and $n_{3}^{\prime \prime \prime}$ in the hypergraphs at the leaf nodes. On the other hand, the original net $n_{5}$ is never split and currently in $\mathcal{H}_{\text {cur }}$. In the figure, without loss of generality, a split net with a single prime in the superscript (e.g., $n_{3}^{\prime}$ ) connects the source vertex of the respective original net $\left(n_{3}\right)$, while split nets with two or more primes (e.g., $\left.n_{3}^{\prime \prime}, n_{3}^{\prime \prime \prime}\right)$ do not.
In the formation of the message nets, we make use of the most recent $\left(2^{\ell}+i\right)$-way partition information $\Pi_{\text {cur }}$. The message nets are categorized into two as send message nets and receive message nets, or simply send nets and receive nets.

We form a send net $s_{k}$ for each $\mathcal{P}_{k} \neq \mathcal{P}_{\text {cur }}$ to which $\mathcal{P}_{\text {cur }}$ sends a message. $s_{k}$ connects the vertices corresponding to the items sent to $\mathcal{P}_{k}$ :

$$
\begin{aligned}
\operatorname{Pins}\left(s_{k}\right)=\left\{v_{j} \in \mathcal{V}_{\text {cur }}:\right. & \operatorname{src}\left(n_{j}\right)=v_{j} \text { and } \\
& \left.\operatorname{Pins}\left(n_{j}\right) \cap \mathcal{V}_{k \neq \text { cur }} \neq \emptyset\right\} .
\end{aligned}
$$

In other words, $s_{k}$ connects source vertex $v_{j}$ of each original net $n_{j}$ that represents the expand operation which necessitates sending $i_{j}$ to $\mathcal{P}_{k}$. Algorithm 1 shows the formation of the set of send nets $\mathcal{N}_{\mathrm{SND}}$, which is initially empty (line 1 ). For each vertex $v_{j} \in \mathcal{V}_{\text {cur }}$, we first retrieve net $n_{j}$ such that $\operatorname{src}\left(n_{j}\right)=v_{j}$ (line 4 ). Then, the vertices which are connected by $n_{j}$ and not in $\mathcal{V}_{\text {cur }}$ are traversed (line 5). Let $v$ be such a vertex, currently in part $\mathcal{V}_{k}$. Since $\mathcal{P}_{k}$ needs $i_{j}$ due to the task 
represented by $v, i_{j}$ is sent from $\mathcal{P}_{\text {cur }}$ to $\mathcal{P}_{k}$. Hence, the vertices connected by send net $s_{k}$ representing this message are updated (lines 7-12): If $s_{k}$ is processed for the first time, $\operatorname{Pins}\left(s_{k}\right)$ is initialized with $\left\{v_{j}\right\}$ and $s_{k}$ is added to $\mathcal{N}_{\mathrm{SND}}$ (lines 8 and 10), otherwise, $\operatorname{Pins}\left(s_{k}\right)$ is updated to include $v_{j}$ (line 12). Since $\mathcal{P}_{\text {cur }}$ can send at most one message to each of $2^{\ell}+i-1$ processor groups, the number of send nets included in $\mathcal{H}_{\text {cur }}^{M}$ is at most $2^{\ell}+i-1$, i.e., $0 \leq\left|\mathcal{N}_{\mathrm{SND}}\right| \leq$ $2^{\ell}+i-1$. In Fig. 5 , the send nets $s_{a}$ and $s_{b}$ are formed and included in $\mathcal{H}_{\text {cur }}^{M}$ to represent the messages from $\mathcal{P}_{\text {cur }}$ to $\mathcal{P}_{a}$ and $\mathcal{P}_{b} . s_{a}$ connects the respective source vertices $v_{1}, v_{2}$ and $v_{3}$ of the original nets $n_{1}, n_{2}$ and $n_{3}$ since $\mathcal{H}_{a}$ contains vertices that are also connected by these nets (indicated by the split nets $n_{1}^{\prime \prime}, n_{2}^{\prime \prime}$ and $n_{3}^{\prime \prime}$ ). Similarly, $s_{b}$ connects $v_{3}$ and $v_{4}$ due to the vertices connected by $n_{3}$ and $n_{4}$ in $\mathcal{H}_{b}$.

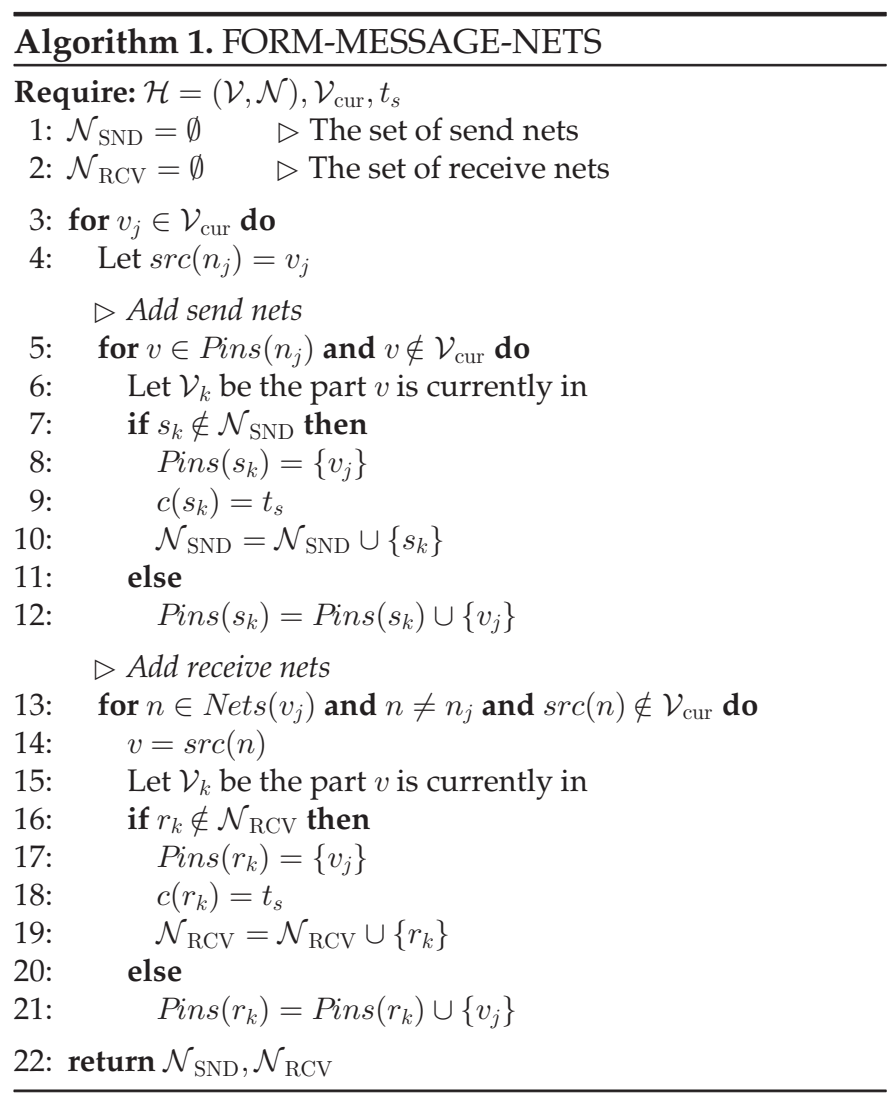

We form a receive net $r_{k}$ for each $\mathcal{P}_{k} \neq \mathcal{P}_{\text {cur }}$ from which $\mathcal{P}_{\text {cur }}$ receives a message. $r_{k}$ connects the vertices corresponding to the tasks that need items from $\mathcal{P}_{k}$ :

$$
\begin{array}{r}
\operatorname{Pins}\left(r_{k}\right)=\left\{v_{j} \in \mathcal{V}_{\text {cur }}: v_{j} \in \operatorname{Pins}(n)\right. \text { and } \\
\left.\operatorname{src}(n) \in \mathcal{V}_{k \neq \text { cur }}\right\} .
\end{array}
$$

In other words, $r_{k}$ connects vertex $v_{j}$ which is connected by each original net $n$ representing the expand operation that necessitates to receive the respective item from $\mathcal{P}_{k}$. Algorithm 1 shows the formation of the set of receive nets $\mathcal{N}_{\mathrm{RCV}}$, which is initially empty (line 2 ). For each vertex $v_{j} \in \mathcal{V}_{\text {cur }}$, we traverse the nets that connect $v_{j}$ other than $n_{j}$ whose source vertices are not in $\mathcal{V}_{\text {cur }}$ (line 13). Let $n$ be such a net and $v$ be its source vertex, currently in $\mathcal{V}_{k}$ (lines 14-15). Since $\mathcal{P}_{\text {cur }}$ needs the item corresponding to $v$ due to task $t_{j}$ represented by $v_{j}$, this item is received by $\mathcal{P}_{\text {cur }}$ from $\mathcal{P}_{k}$. Hence, the vertices connected by receive net $r_{k}$ representing this message are updated (lines 16-21): If $r_{k}$ is processed for the first time, Pins $\left(r_{k}\right)$ is initialized with $\left\{v_{j}\right\}$ and $r_{k}$ is added to $\mathcal{N}_{\mathrm{RCV}}$ (lines 17 and 19), otherwise, $\operatorname{Pins}\left(r_{k}\right)$ is updated to include $v_{j}$ (line 21). Since $\mathcal{P}_{\text {cur }}$ can receive at most one message from each of the $2^{\ell}+i-1$ processor groups, the number of receive nets included in $\mathcal{H}_{\text {cur }}^{M}$ is at most $2^{\ell}+i-1$, i.e., $0 \leq\left|\mathcal{N}_{\mathrm{RCV}}\right| \leq$ $2^{\ell}+i-1$. In Fig. 5, the receive nets $r_{b}$ and $r_{c}$ are formed and included in $\mathcal{H}_{\text {cur }}^{M}$ to represent the messages from $\mathcal{P}_{b}$ and $\mathcal{P}_{c}$ to $\mathcal{P}_{\text {cur }} . r_{b}$ connects $v_{1}$ and $v_{2}$, which are connected by $n_{6}$ (indicated by the split net $\left.n_{6}^{\prime \prime}\right)$, since $\mathcal{H}_{b}$ contains the source vertex of $n_{6}$. Similarly, $r_{c}$ connects $v_{2}, v_{4}$ and $v_{5}$ due to the nets $n_{7}$ and $n_{8}$, both of which have their source vertices in $\mathcal{H}_{c}$.

Recall that the cost of a volume net in $\mathcal{H}_{\text {cur }}$ is $\operatorname{size}\left(i_{j}\right) t_{w}$ and captures the bandwidth cost. To capture the latency cost via message nets, the costs of these nets are assigned the startup latency:

$$
c\left(s_{k}\right)=c\left(r_{k}\right)=t_{s}, \quad \text { for } s_{k} \in \mathcal{N}_{\mathrm{SND}} \text { and } r_{k} \in \mathcal{N}_{\mathrm{RCV}},
$$

in lines 9 and 18 of Algorithm 1. Note that the cost of a volume net is the size of the corresponding item in terms of $t_{w}$, whereas the cost of a message net is unit in terms of $t_{s}$ since it encapsulates exactly one message. The message nets have a higher cost than the volume nets since the startup time of a message is significantly higher than the time required to transmit a word. Finally, the message nets in $\mathcal{N}_{\text {SND }}$ and $\mathcal{N}_{\text {RCV }}$ are returned (line 22).

\subsubsection{Partitioning and Correctness}

The newly formed hypergraph $\mathcal{H}_{\text {cur }}^{M}$ is then bipartitioned to obtain $\Pi\left(\mathcal{H}_{\text {cur }}^{M}\right)=\left\{\mathcal{V}_{L}, \mathcal{V}_{R}\right\}$. Maintaining balance in partitioning $\mathcal{H}_{\text {cur }}^{M}$ is the same with that of $\mathcal{H}_{\text {cur }}$ since vertices and their weights are the same in both hypergraphs. With the newly introduced message nets, the cutsize of $\Pi$ is given by

$$
\begin{aligned}
\operatorname{cut}(\Pi) & =\sum_{n_{j} \in \mathcal{N}_{\text {cur }}^{\text {cut }}} c\left(n_{j}\right)+\sum_{s_{k} \in \mathcal{N}_{\mathrm{SND}}^{\text {cut }}} c\left(s_{k}\right)+\sum_{r_{k} \in \mathcal{N}_{\mathrm{RCV}}^{\text {cut }}} c\left(r_{k}\right) \\
& =\sum_{n_{j} \in \mathcal{N}_{\text {cur }}^{\text {cut }}} \operatorname{size}\left(i_{j}\right) t_{w}+\left|\mathcal{N}_{\mathrm{SND}}^{\text {cut }}\right| t_{s}+\left|\mathcal{N}_{\mathrm{RCV}}^{\text {cut }}\right| t_{s},
\end{aligned}
$$

where $\mathcal{N}_{\text {cur }}^{\text {cut }}, \mathcal{N}_{\mathrm{SND}}^{\text {cut }}$ and $\mathcal{N}_{\mathrm{RCV}}^{\text {cut }}$ respectively denote the sets of cut volume nets, cut send nets and cut receive nets in $\Pi\left(\mathcal{H}_{\text {cur }}^{M}\right)$.

Let $m s g(\mathbb{P})$ denote the total number of messages communicated among the processor groups in $\mathbb{P}$.

Theorem 1. Consider an RB tree prior to bipartitioning the ith hypergraph $\mathcal{H}_{\text {cur }}^{M}=\left(\mathcal{V}_{\text {cur }}, \mathcal{N}_{\text {cur }}^{M}\right)$ in the lth level with message nets added. The vertex sets of the leaf hypergraphs of the RB tree are assumed to induce a $\left(2^{\ell}+i\right)$-way processor partition $\mathbb{P}_{\text {cur. }}$. Suppose that the bipartition $\Pi\left(\mathcal{H}_{\text {cur }}^{M}\right)=\left\{\mathcal{V}_{L}, \mathcal{V}_{R}\right\}$ generates two new leaf hypergraphs $\mathcal{H}_{L}$ and $\mathcal{H}_{R}$, where processor groups $\mathcal{P}_{L}$ and $\mathcal{P}_{R}$ are associated with $\mathcal{V}_{L}$ and $\mathcal{V}_{R}$. After bipartitioning, the vertex sets of the hypergraphs are assumed to induce a $\left(2^{\ell}+i+1\right)$-way processor partition $\mathbb{P}_{\text {new }}=\mathbb{P}_{\text {cur }}-$ $\left\{\mathcal{P}_{\text {cur }}\right\} \cup\left\{\mathcal{P}_{L}, \mathcal{P}_{R}\right\}$. Minimizing the number of cut message nets 
in $\Pi\left(\mathcal{H}_{\text {cur }}^{M}\right)$ minimizes the increase $\Delta M$ in the number of messages between $\mathcal{P}_{\text {cur }}$ and $\mathbb{P}_{\text {cur }}-\left\{\mathcal{P}_{\text {cur }}\right\}$, which is given by

$$
\Delta M=m s g\left(\mathbb{P}_{\text {new }}\right)-m s g\left(\mathbb{P}_{\text {cur }}\right)-m s g\left(\left\{\mathcal{P}_{L}, \mathcal{P}_{R}\right\}\right),
$$

where $\operatorname{msg}\left(\left\{\mathcal{P}_{L}, \mathcal{P}_{R}\right\}\right) \in\{0,1,2\}$.

Proof. A send net $s_{k}$ in $\mathcal{H}_{\text {cur }}^{M}$ signifies a message from $\mathcal{P}_{\text {cur }}$ to $\mathcal{P}_{k} \in \mathbb{P}_{\text {cur }}-\left\{\mathcal{P}_{\text {cur }}\right\}$. If $s_{k}$ is a cut-net in $\Pi\left(\mathcal{H}_{\text {cur }}^{M}\right)$, then both $\mathcal{P}_{L}$ and $\mathcal{P}_{R}$ send a message to $\mathcal{P}_{k}$. The message from $\mathcal{P}_{L}$ to $\mathcal{P}_{k}$ and the message from $P_{R}$ to $P_{k}$ respectively contain the items corresponding to the vertices in $\operatorname{Pins}\left(s_{k}\right) \cap \mathcal{V}_{L}$ and $\operatorname{Pins}\left(s_{k}\right) \cap \mathcal{V}_{R}$. Hence, a cut send net contributes one to $\Delta M$. If $s_{k}$ is uncut being in either $\mathcal{V}_{L}$ or $\mathcal{V}_{R}$, then only the respective processor group sends a message to $\mathcal{P}_{k}$, whose content is exactly the same with the message from $\mathcal{P}_{\text {cur }}$ to $\mathcal{P}_{k}$. Hence, an uncut send net does not contribute to $\Delta M$.

In a dual manner, a receive net $r_{k}$ in $\mathcal{H}_{\text {cur }}^{M}$ signifies a message from $\mathcal{P}_{k} \in \mathbb{P}_{\text {cur }}-\left\{\mathcal{P}_{\text {cur }}\right\}$ to $\mathcal{P}_{\text {cur }}$. If $r_{k}$ is a cut-net in $\Pi\left(\mathcal{H}_{\text {cur }}^{M}\right)$, then both $\mathcal{P}_{L}$ and $\mathcal{P}_{R}$ receive a message from $\mathcal{P}_{k}$. The message from $\mathcal{P}_{k}$ to $\mathcal{P}_{L}$ and the message from $\mathcal{P}_{k}$ to $\mathcal{P}_{R}$ respectively contain the items required by the tasks corresponding to the vertices in $\operatorname{Pins}\left(r_{k}\right) \cap \mathcal{V}_{L}$ and $\operatorname{Pins}\left(r_{k}\right) \cap \mathcal{V}_{R}$. Hence, a cut receive net also contributes one to $\Delta M$. If $r_{k}$ is uncut being in either $\mathcal{V}_{L}$ or $\mathcal{V}_{R}$, then only the respective processor group receives a message from $\mathcal{P}_{k}$, whose content is exactly the same with the message from $\mathcal{P}_{k}$ to $\mathcal{P}_{\text {cur }}$. Hence, an uncut receive net does not contribute to $\Delta M$.

The message nets are oblivious to the messages between $\mathcal{P}_{L}$ and $\mathcal{P}_{R}$ since our approach introduces these message nets for the processor groups in $\mathbb{P}_{\text {cur. }}$. For this reason, $\operatorname{msg}\left(\left\{\mathcal{P}_{L}, \mathcal{P}_{R}\right\}\right)$ is not taken into account. Therefore, $\Delta M$ is equal to the number of cut message nets.

By Theorem 1, the number of cut message nets in $\Pi\left(\mathcal{H}_{\text {cur }}^{M}\right),\left|\mathcal{N}_{\text {SND }}^{\text {cut }}\right|+\left|\mathcal{N}_{\mathrm{RCV}}^{\text {cut }}\right|$, is equal to the increase in the message count, where the new messages between $\mathcal{P}_{L}$ and $\mathcal{P}_{R}$ are excluded. In other words, the number of cut message nets corresponds to the increase in the number of messages between $\mathcal{P}_{\text {cur }}$ and $\mathbb{P}_{\text {cur }}-\left\{\mathcal{P}_{\text {cur }}\right\}$ with $\mathcal{P}_{\text {cur }}$ bipartitioned into $\left\{\mathcal{P}_{L}, \mathcal{P}_{R}\right\}$ in $\mathbb{P}_{\text {new }}$. Observe that each cut message net contributes its associated cost $c\left(s_{k}\right)$ or $c\left(r_{k}\right)$ to the cutsize (6). For this reason as well as because of the presence of volume nets in (6), minimizing the cutsize does not exactly correspond to but relates to minimizing the number of cut message nets. Considering both the volume and the message nets, minimizing the cutsize corresponds to reducing both the total volume and the total message count.

Partitioning $\mathcal{H}_{\text {cur }}^{M}$ is oblivious to the messages between $\mathcal{P}_{L}$ and $\mathcal{P}_{R}$. However, $\operatorname{msg}\left(\left\{\mathcal{P}_{L}, \mathcal{P}_{R}\right\}\right)$ is negligible compared to $m s g\left(\mathbb{P}_{\text {new }}\right)-m s g\left(\mathbb{P}_{\text {cur }}\right)$ since it is upper bounded by two. Moreover, $\operatorname{msg}\left(\left\{\mathcal{P}_{L}, \mathcal{P}_{R}\right\}\right)$ is empirically found to be almost constant as 2, being 0 or 1 in only 0.1 percent of $1 \mathrm{M}$ bipartitions. Note that $0 \leq \Delta M \leq 2\left(2^{\ell}+i-1\right)$. The worst case for $\Delta M$ occurs when $\mathcal{H}_{\text {cur }}^{M}$ contains a send and a receive net for each other processor group in $\mathbb{P}_{\text {cur }}$ and they all become cut in $\Pi\left(\mathcal{H}_{\text {cur }}^{M}\right)$.

In Fig. 5, there are two send nets $s_{a}$ and $s_{b}$ and two receive nets $r_{b}$ and $r_{c}$ in $\mathcal{H}_{\text {cur. }}^{M}$. Among the send nets, $s_{a}$ is a cut-net whereas $s_{b}$ is an uncut net in $\mathcal{H}_{R}$ after bipartitioning. $s_{a}$ necessitates both $\mathcal{P}_{L}$ and $\mathcal{P}_{R}$ to send a message to $\mathcal{P}_{a}$ due to the items $\left\{i_{1}, i_{2}\right\}$ and $\left\{i_{3}\right\}$, respectively. Since $s_{a}$ is a cutnet, it increases the total message count by one as $\mathcal{P}_{\text {cur }}$ was sending a message to $\mathcal{P}_{a} . s_{b}$ necessitates only $\mathcal{P}_{R}$ to send a message to $\mathcal{P}_{b}$ due to the items $\left\{i_{3}, i_{4}\right\}$. Since $s_{b}$ is an uncut net, it does not change the total message count as $\mathcal{P}_{\text {cur }}$ was already sending a message to $\mathcal{P}_{b}$. Among the receive nets, $r_{c}$ is a cut-net whereas $r_{b}$ is an uncut net in $\mathcal{H}_{L}$ after bipartitioning. $r_{c}$ necessitates both $\mathcal{P}_{L}$ and $\mathcal{P}_{R}$ to receive a message from $\mathcal{P}_{c}$ due to the tasks $\left\{t_{2}\right\}$ and $\left\{t_{4}, t_{5}\right\}$, respectively. Since $r_{c}$ is a cut-net, it increases the total message count by one as $\mathcal{P}_{\text {cur }}$ was receiving a message from $\mathcal{P}_{c}$. $r_{b}$ necessitates only $\mathcal{P}_{L}$ to receive a message from $\mathcal{P}_{b}$ due to the tasks $\left\{t_{1}, t_{2}\right\}$. Since $r_{b}$ is an uncut net, it does not change the total message count as $\mathcal{P}_{\text {cur }}$ was already receiving a message from $\mathcal{P}_{b}$. Hence, two cut message nets cause an increase of two in the number of messages between $\mathcal{P}_{\text {cur }}$ and the other processor groups.

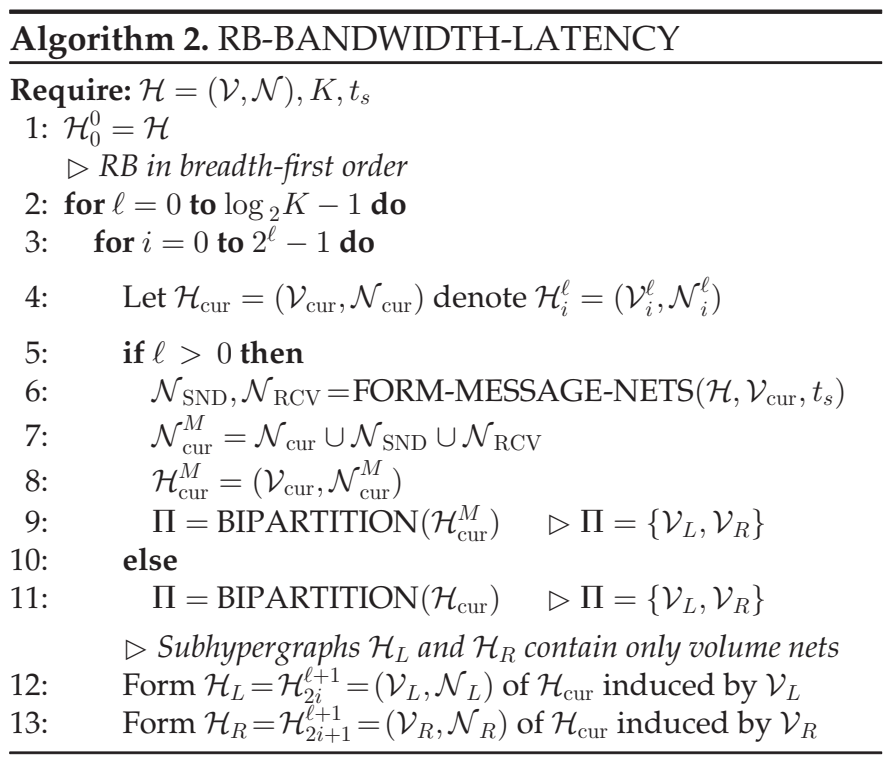

Algorithm 2 displays the overall RB process in which both the bandwidth and the latency costs are reduced. As inputs, the algorithm takes a hypergraph $\mathcal{H}=(\mathcal{V}, \mathcal{N}), K$ (the number of parts to be obtained), and $t_{s}$ as the cost of the message nets. The partitioning proceeds in a breadth-first manner (lines 2-3). Each hypergraph $\mathcal{H}_{\text {cur }}$ to be bipartitioned does not contain message nets initially (line 4). The sets of send and receive nets $\mathcal{N}_{\mathrm{SND}}$ and $\mathcal{N}_{\mathrm{RCV}}$ are formed via FORM-MESSAGE-NETS (Algorithm 1) (line 6). Then, these message nets are added to $\mathcal{N}_{\text {cur }}$ to obtain $\mathcal{N}_{\text {cur }}^{M}$ (line 7) and consequently $\mathcal{H}_{\text {cur }}^{M}$ (line 8 ). Note that if $\mathcal{H}_{\text {cur }}$ is the root hypergraph $\mathcal{H}_{0}^{0}$ of the RB tree, no message nets can be added since there is only a single processor group at this point (line 10). The current hypergraph is bipartitioned with the BIPARTITION function to obtain the vertex parts $\mathcal{V}_{L}$ and $\mathcal{V}_{R}$ (lines 9 and 11). The call to BIPARTITION can be realized with any hypergraph partitioning tool; it is a call to obtain only a two-way partition. New hypergraphs $\mathcal{H}_{L}=\mathcal{H}_{2 i}^{\ell+1}$ and $\mathcal{H}_{R}=\mathcal{H}_{2 i+1}^{\ell+1}$ are formed as described in Section 2.2 (lines 1213). $\mathcal{N}_{L}$ and $\mathcal{N}_{R}$ do not contain any message nets since these nets rely on the most recent partitioning information and thus need to be introduced from scratch just prior to bipartitioning $\mathcal{H}_{2 i}^{\ell+1}$ and $\mathcal{H}_{2 i+1}^{\ell+1}$. Notice that at any step of the RB, 
among all the leaf hypergraphs, only the current $\mathcal{H}_{\text {cur }}$ is subject to the addition of the message nets, whereas other hypergraphs remain intact.

\subsubsection{Running Time Analysis}

We consider the cost of adding message nets in the $\ell$ th level of the RB tree produced in partitioning $\mathcal{H}$ into $K$ parts, $0<\ell<\log _{2} K$. Recall that Algorithm 1 utilizes Pins $(\cdot)$ and $\operatorname{Nets}(\cdot)$ functions on the original hypergraph $\mathcal{H}=(\mathcal{V}, \mathcal{N})$.

In the addition of the send nets, for each vertex $v_{j}$ in the $\ell$ th level, $\operatorname{Pins}\left(n_{j}\right)$ is visited once, where $\operatorname{src}\left(n_{j}\right)=v_{j}$ (line 5 in Algorithm 1). Observe that each net in $\mathcal{N}$ is visited only once since $n_{j}$ is retrieved only for $v_{j}$. Updates related to a send net (lines 7-12) can be performed in $O(1)$ time. Hence, each pin of $\mathcal{H}$ is processed exactly once, making the cost of formation and addition of send nets $O(p)$ in the $\ell$ th level, where $p$ is the number of pins in $\mathcal{H}$.

In the addition of the receive nets, for each vertex $v_{j}$ in the $\ell$ th level, Nets $\left(v_{j}\right)$ is visited once (line 13 in Algorithm 1). Updates related to a receive net (lines 16-21) can also be performed in $O(1)$ time. Hence, each pin of $\mathcal{H}$ is again processed exactly once, making the cost of formation and addition of receive nets $O(p)$ in the $\ell$ th level.

Therefore, the cost of adding message nets in a single level of RB is $O(p)$, which results in the overall cost of $O\left(p \log _{2} K\right)$ for adding message nets. The solution of the partitioning problem with the addition of message nets is likely to be more expensive compared to partitioning of the original hypergraph.

\subsection{Extensions}

\subsubsection{Encoding Messages for $A_{P O S T}$}

We now describe how to apply the proposed model to $A_{P O S T}$. In $\mathcal{H}_{F}$, we define a function dest: $\mathcal{N} \rightarrow \mathcal{V}$ to determine the responsibility of the fold operation on each $o_{j}$, similar to the definition of $\operatorname{src}$ for expand operations in $\mathcal{H}_{E}$. In partitioning $\mathcal{H}_{F}$, a send net $s_{k}$ connects the vertices corresponding to the tasks that produce intermediate results to be sent to $\mathcal{P}_{k}$ :

$$
\begin{array}{r}
\operatorname{Pins}\left(s_{k}\right)=\left\{v_{j} \in \mathcal{V}_{\text {cur }}:\right. \\
v_{j} \in \operatorname{Pins}(n) \text { and } \\
\left.\operatorname{dest}(n) \in \mathcal{V}_{k \neq \text { cur }}\right\} .
\end{array}
$$

A receive net $r_{k}$ connects the vertices corresponding to the output items for which the intermediate results need to be received from $\mathcal{P}_{k}$ :

$$
\begin{aligned}
\operatorname{Pins}\left(r_{k}\right)=\left\{v_{j} \in \mathcal{V}_{\text {cur }}:\right. & \operatorname{dest}\left(n_{j}\right)=v_{j} \text { and } \\
& \left.\operatorname{Pins}\left(n_{j}\right) \cap \mathcal{V}_{k \neq \text { cur }} \neq \emptyset\right\} .
\end{aligned}
$$

Observe that the formation of a send net for $\mathcal{H}_{F}$ (8) is the same as that of a receive net for $\mathcal{H}_{E}$ (4) and the formation of a receive net for $\mathcal{H}_{F}(9)$ is the same as that of a send net for $\mathcal{H}_{E}$ (3). So, the message nets in $\mathcal{H}_{E}$ are the dual of the message nets in $\mathcal{H}_{F}$. Therefore, the correctness and complexity analysis carried out for $A_{P R E}$ are also valid for $A_{P O S T}$.

\subsubsection{Encoding Messages for $I=O \geq T$}

To extend the proposed model to the case $I=O \geq T$ for

\begin{tabular}{|c|c|c|c|}
\hline name & kind & \#rows/cols & \#nonzeros \\
\hline d_pretok & $2 \mathrm{D} / 3 \mathrm{D}$ & 182,730 & $1,641,672$ \\
\hline turon_m & $2 \mathrm{D} / 3 \mathrm{D}$ & 189,924 & $1,690,876$ \\
\hline cop20k_A & $2 \mathrm{D} / 3 \mathrm{D}$ & 121,192 & $2,624,331$ \\
\hline torso3 & $2 \mathrm{D} / 3 \mathrm{D}$ & 259,156 & $4,429,042$ \\
\hline mono_500Hz & acoustics & 169,410 & $5,036,288$ \\
\hline memchip & circuit sim. & $2,707,524$ & $14,810,202$ \\
\hline Freescale1 & circuit sim. & $3,428,755$ & $18,920,347$ \\
\hline circuit5M_dc & circuit sim. & $3,523,317$ & $19,194,193$ \\
\hline rajat31 & circuit sim. & $4,690,002$ & $20,316,253$ \\
\hline laminar_duct3D & comp. fluid dyn. & 67,173 & $3,833,077$ \\
\hline StocF-1465 & comp. fluid dyn. & $1,465,137$ & $21,005,389$ \\
\hline web-Google & directed graph & 916,428 & $5,105,039$ \\
\hline in-2004 & directed graph & $1,382,908$ & $16,917,053$ \\
\hline eu-2005 & directed graph & 862,664 & $19,235,140$ \\
\hline cage14 & directed graph & $1,505,785$ & $27,130,349$ \\
\hline mac_econ_fwd500 & economic & 206,500 & $1,273,389$ \\
\hline gsm_106857 & electromagnetics & 589,446 & $21,758,924$ \\
\hline pre2 & freq.-dom. circuit sim. & 659,033 & $5,959,282$ \\
\hline kkt_power & optimization & $2,063,494$ & $14,612,663$ \\
\hline bcsstk31 & structural & 35,588 & $1,181,416$ \\
\hline engine & structural & 143,571 & $4,706,073$ \\
\hline shipsec 8 & structural & 114,919 & $6,653,399$ \\
\hline Transport & structural & $1,602,111$ & $23,500,731$ \\
\hline $\mathrm{CO}$ & theor./quant. chem. & 221,119 & $7,666,057$ \\
\hline $598 a$ & undirected graph & 110,971 & $1,483,868$ \\
\hline $\mathrm{m} 14 \mathrm{~b}$ & undirected graph & 214,765 & $3,358,036$ \\
\hline roadNet-CA & undirected graph & $1,971,281$ & $5,533,214$ \\
\hline great-britain_osm & undirected graph & $7,733,822$ & $16,313,034$ \\
\hline germany_osm & undirected graph & $11,548,845$ & $24,738,362$ \\
\hline debr & undirected graph seq. & $1,048,576$ & $4,194,298$ \\
\hline
\end{tabular}
$A_{P R E}$, we need a minor change in the formation of the
TABLE 1

Properties of Test Matrices

message nets. In this case, a net $n_{j}$ might be held responsible for multiple expand operations (see src definition). To reflect this change, line 4 of Algorithm 1 needs to be executed for each net $n_{j}$ such that $\operatorname{src}\left(n_{j}\right)=v_{j}$. The meaning of a message net does not change. The complexity of adding message nets is still $O\left(p \log _{2} K\right)$ since each net $n_{j}$ is again retrieved exactly once, uniquely by $v_{j}$. A similar discussion holds for $A_{P O S T}$ by extending the definition of dest.

\section{EXPERIMENTS}

\subsection{Setup}

For evaluation, we target the parallelization of an $A_{P R E}$ application: 1D row-parallel SpMV. We model this application with hypergraph $\mathcal{H}_{E}$ as described in Section 2.3. We compare two schemes for the partitioning of $\mathcal{H}_{E}$ :

- HP: The standard hypergraph partitioning model in which only the bandwidth cost is minimized (Section 2.3). In this scheme, $\mathcal{H}_{E}$ contains only the volume nets.

- HP-L: The proposed hypergraph partitioning model in which the bandwidth and the latency costs are reduced simultaneously (Section 3). In this scheme, $\mathcal{H}_{E}$ contains both the volume and the message nets.

Both HP and HP-L utilize recursive bipartitioning. We tested these schemes for $K \in\{128,256,512,1024,2048\}$ processors.

The two schemes are evaluated on 30 square matrices from the UFL Sparse Matrix Collection [31]. Table 1 displays the properties of these matrices. We consider square 
matrices since the proposed scheme aims at obtaining a conformal partition on the input and output items. The numbers of nonzeros in the test matrices range from $1.2 \mathrm{M}$ to $27.1 \mathrm{M}$. This dataset contains small matrices (e.g., bcsstk31, mac_econ_fwd500, etc.) for which the latency cost is expected to be more important.

The partitionings for both HP and HP-L are performed with the hypergraph partitioner PaToH [1]. Specifically, for each bipartitioning, we call PaToH_Part function of PaToH (lines 7 and 9 of Algorithm 2). The partitioning imbalance is set to 10 percent. Since $\mathrm{PaToH}$ contains randomized algorithms, we run each partitioning instance five times and report the average results of these runs.

We present the communication statistics for partitionings obtained via HP and HP-L and the corresponding parallel SpMV times. We used parallel SpMV of PETSc toolkit [32] on a Blue Gene/Q system. A node on this system consists of 16 PowerPC A2 processors with $1.6 \mathrm{GHz}$ clock frequency and 16 GB memory. The nodes are connected by a 5D torus chip-to-chip network.

\subsection{Message Net Costs}

Recall that in our model, the volume nets are assigned the cost of $t_{w}$ (transfer time of a single word) and the message nets are assigned the cost of $t_{s}$ (startup time). Hereinafter, both the bandwidth and the latency costs are expressed in terms of $t_{w}$ for the sake of presentation. Hence, the costs of volume nets are unit whereas the costs of message nets are $t_{s} / t_{w}$. The message net costs are denoted with mnc to simplify the notation. We conducted ping-pong experiments on BlueGene/Q with varying message sizes and the average $t_{s} / t_{w}$ ratio was found to be around 200 for the matrices in our dataset.

Compared to HP, HP-L is expected to obtain a higher total volume since HP-L addresses two communication components simultaneously while HP solely optimizes a single component, which is determined by the total volume. We found out that the cost assignment of message nets in HP-L has a crucial impact on the parallel performance. The $t_{s} / t_{w}$ ratio varies in practice for different message sizes and depends on the protocol used for transmitting messages as well as the characteristics of the target application which is likely to incur a higher $t_{w}$, hence a lower $t_{s} / t_{w}$, than that was found. For this reason, as well as to control the balance between the increase in the volume and the decrease in the message count compared to $\mathrm{HP}$, we tried out different values for $m n c$ in HP-L. The tested $m n c$ values are 10, 50, 100 and 200. The reason for including smaller $m n c$ values is that when the communication cost is dominated by the bandwidth component, utilizing a high $m n c$ value has an adverse affect on the parallel performance compared to HP as the volume increase caused by HP-L is more apparent. Hence, small $m n c$ values become more preferable in such cases.

\subsection{Results}

Table 2 presents the average communication statistics and the parallel SpMV running times of HP-L normalized with respect to those of HP for four $m n c$ values and five $K$ values. Each entry at a specific $K$ value is the geometric mean of the normalized results obtained at that $K$ value. The communication statistics are grouped under "volume" and
TABLE 2

Communication Statistics, PaToH Partitioning Times and Parallel SpMV Running Times for HP-L Normalized with Respect to those for HP Averaged over 30 Matrices

\begin{tabular}{|c|c|c|c|c|c|c|c|}
\hline \multirow{2}{*}{$\begin{array}{l}\text { message } \\
\text { net } \\
\text { cost }\end{array}$} & \multirow[b]{2}{*}{$K$} & \multicolumn{2}{|c|}{ volume } & \multicolumn{2}{|c|}{ \#messages } & \multirow{2}{*}{$\begin{array}{c}\text { PaToH } \\
\text { part. } \\
\text { time }\end{array}$} & \multirow{2}{*}{$\begin{array}{c}\text { parallel } \\
\text { SpMV } \\
\text { time }\end{array}$} \\
\hline & & tot & $\max$ & tot & $\max$ & & \\
\hline \multirow{5}{*}{10} & 128 & 1.08 & 1.11 & 0.82 & 0.87 & 1.07 & 0.956 \\
\hline & 256 & 1.10 & 1.16 & 0.78 & 0.83 & 1.13 & 0.904 \\
\hline & 512 & 1.12 & 1.22 & 0.75 & 0.83 & 1.13 & 0.838 \\
\hline & 1024 & 1.16 & 1.29 & 0.73 & 0.84 & 1.25 & 0.792 \\
\hline & 2048 & 1.20 & 1.37 & 0.71 & 0.88 & 1.28 & 0.774 \\
\hline \multirow{5}{*}{50} & 128 & 1.17 & 1.25 & 0.65 & 0.76 & 1.08 & 0.924 \\
\hline & 256 & 1.25 & 1.44 & 0.59 & 0.70 & 1.14 & 0.846 \\
\hline & 512 & 1.33 & 1.57 & 0.56 & 0.69 & 1.21 & 0.760 \\
\hline & 1024 & 1.41 & 1.69 & 0.57 & 0.74 & 1.24 & 0.715 \\
\hline & 2048 & 1.48 & 1.85 & 0.59 & 0.80 & 1.33 & 0.708 \\
\hline \multirow{5}{*}{100} & 128 & 1.24 & 1.43 & 0.59 & 0.73 & 1.09 & 0.954 \\
\hline & 256 & 1.35 & 1.66 & 0.53 & 0.68 & 1.17 & 0.858 \\
\hline & 512 & 1.45 & 1.86 & 0.51 & 0.68 & 1.19 & 0.768 \\
\hline & 1024 & 1.54 & 1.92 & 0.53 & 0.71 & 1.31 & 0.706 \\
\hline & 2048 & 1.61 & 2.06 & 0.57 & 0.80 & 1.41 & 0.707 \\
\hline \multirow{5}{*}{200} & 128 & 1.33 & 1.60 & 0.54 & 0.72 & 1.15 & 1.031 \\
\hline & 256 & 1.46 & 1.87 & 0.48 & 0.67 & 1.19 & 0.872 \\
\hline & 512 & 1.57 & 2.02 & 0.49 & 0.67 & 1.25 & 0.778 \\
\hline & 1024 & 1.65 & 2.09 & 0.52 & 0.72 & 1.37 & 0.722 \\
\hline & 2048 & 1.70 & 2.17 & 0.57 & 0.79 & 1.48 & 0.712 \\
\hline
\end{tabular}

"\#messages". Under the "volume" grouping, the column "tot" denotes the total volume of communication and the column "max" denotes the maximum send volume of processors. Under the "\#messages" grouping, the column "tot" denotes the total number of messages and the column "max" denotes the maximum number of messages that a processor sends. The PaToH partitioning times and parallel SpMV running times are respectively given under the columns "PaToH part. time" and "parallel SpMV time".

As seen in Table 2, HP-L achieves a significant reduction in the latency overhead. HP-L reduces the total number of messages by 18-29 percent, 35-44 percent, 41-49 percent and 43-52 percent for $m n c$ values of 10, 50, 100 and 200, respectively, compared to HP. This substantial improvement comes at the expense of increased volume as expected. Compared to HP, HP-L increases the total volume by 8-20 percent, 17-48 percent, 24-61 percent and 33-70 percent for mnc values of 10, 50, 100 and 200, respectively. In other words, HP-L achieves a factor of 1.22-1.41, 1.54-1.79, 1.69-1.96 and 1.75-2.08 reductions in the total number of messages while causing a factor of 1.08-1.20, 1.17-1.48, 1.24-1.61 and 1.33-1.70 increase in the total volume, over HP for $m n c$ values of 10,50, 100 and 200, respectively.

The proposed HP-L scheme achieves significantly lower parallel SpMV running times compared to the HP scheme. As seen in Table 2, HP-L achieves 4-23 percent, 8-29 percent, 5-29 percent and -3-29 percent lower running times for $m n c$ values of 10, 50, 100 and 200, respectively, compared to HP. The lowest average running times for $K$ values of 128, 256, 512, 1024 and 2048 are obtained with $m n c$ values of 50, 50, 50, 100 and 100, respectively. Since using a low mnc (e.g., 10) does not attribute enough importance to the reduction of the latency cost, the parallel running times attained by 
TABLE 3

Communication Statistics and Parallel SpMV Times/Speedups for HP and HP-L for $K=512$ and $m n c=50$

\begin{tabular}{|c|c|c|c|c|c|c|c|c|c|c|c|c|}
\hline \multirow[b]{2}{*}{ name } & \multicolumn{2}{|c|}{ tot vol } & \multicolumn{2}{|c|}{ max vol } & \multicolumn{2}{|c|}{ tot msg } & \multicolumn{2}{|c|}{$\max \operatorname{msg}$} & \multicolumn{2}{|c|}{ running time } & \multicolumn{2}{|c|}{ speedup } \\
\hline & $\mathrm{HP}$ & HP-L & $\mathrm{HP}$ & HP-L & HP & HP-L & $\mathrm{HP}$ & HP-L & $\mathrm{HP}$ & HP-L & $\mathrm{HP}$ & HP-L \\
\hline d_pretok & $108 \mathrm{k}$ & $141 k$ & 302 & 451 & $5.3 \mathrm{k}$ & $2.8 \mathrm{k}$ & 19 & 11 & 312 & 232 & 66 & 88 \\
\hline turon_m & $106 \mathrm{k}$ & $127 \mathrm{k}$ & 296 & 434 & $4.8 \mathrm{k}$ & $2.5 \mathrm{k}$ & 16 & 9 & 268 & 210 & 79 & 100 \\
\hline cop20k_A & $142 \mathrm{k}$ & $167 \mathrm{k}$ & 411 & 482 & $5.2 \mathrm{k}$ & $3.6 \mathrm{k}$ & 20 & 15 & 542 & 466 & 82 & 95 \\
\hline torso3 & $197 \mathrm{k}$ & $227 \mathrm{k}$ & 550 & 825 & $4.9 \mathrm{k}$ & $3.2 \mathrm{k}$ & 21 & 15 & 411 & 348 & 112 & 132 \\
\hline mono $500 \mathrm{~Hz}$ & $226 \mathrm{k}$ & $255 \mathrm{k}$ & 703 & 812 & $6.0 \mathrm{k}$ & $4.7 \mathrm{k}$ & 26 & 26 & 492 & 498 & 158 & 157 \\
\hline memchip & $102 \mathrm{k}$ & $149 \mathrm{k}$ & 679 & 705 & $3.9 \mathrm{k}$ & $2.2 \mathrm{k}$ & 51 & 16 & 1,224 & 714 & 188 & 322 \\
\hline Freescale1 & $85 \mathrm{k}$ & $151 k$ & 473 & 972 & $5.4 \mathrm{k}$ & $2.4 \mathrm{k}$ & 68 & 28 & 1,697 & 1,105 & 176 & 271 \\
\hline circuit5M_dc & $84 \mathrm{k}$ & $145 \mathrm{k}$ & 386 & 939 & $5.5 \mathrm{k}$ & $2.3 \mathrm{k}$ & 64 & 24 & 1,554 & 1,030 & 196 & 295 \\
\hline rajat31 & $139 \mathrm{k}$ & $184 \mathrm{k}$ & 403 & 786 & $2.9 \mathrm{k}$ & $2.0 \mathrm{k}$ & 23 & 20 & 1,010 & 983 & 325 & 334 \\
\hline minar_duct3D & $186 \mathrm{k}$ & $211 \mathrm{k}$ & 538 & 694 & $7.4 \mathrm{k}$ & $5.9 \mathrm{k}$ & 26 & 21 & 445 & 387 & 74 & 85 \\
\hline tocF-1465 & $581 \mathrm{k}$ & $613 \mathrm{k}$ & 1650 & 2109 & $6.8 \mathrm{k}$ & $5.5 \mathrm{k}$ & 31 & 29 & 1,062 & 1,048 & 229 & 233 \\
\hline veb-Google & $126 \mathrm{k}$ & $266 \mathrm{k}$ & 872 & 2971 & $37.2 \mathrm{k}$ & $11.9 \mathrm{k}$ & 281 & 231 & 17,385 & 3,492 & 12 & 61 \\
\hline in-2004 & $122 \mathrm{k}$ & $169 \mathrm{k}$ & 1,933 & 2,641 & $12.8 \mathrm{k}$ & $6.4 \mathrm{k}$ & 137 & 181 & 15,690 & 4,164 & 17 & 64 \\
\hline $\mathrm{u}-2005$ & $338 \mathrm{k}$ & $408 \mathrm{k}$ & 4,207 & 6,934 & $18.9 \mathrm{k}$ & $9.9 \mathrm{k}$ & 305 & 351 & 8,375 & 4,849 & 26 & 44 \\
\hline cage14 & $3,184 \mathrm{k}$ & $3,291 \mathrm{k}$ & 10,528 & 10,922 & $27.4 \mathrm{k}$ & $19.7 \mathrm{k}$ & 125 & 100 & 5,757 & 4,587 & 57 & 71 \\
\hline mac_econ_fwd500 & $124 \mathrm{k}$ & $160 \mathrm{k}$ & 335 & 490 & $5.6 \mathrm{k}$ & $3.1 \mathrm{k}$ & 16 & 11 & 252 & 231 & 80 & 88 \\
\hline gsm 106857 & $338 \mathrm{k}$ & $371 \mathrm{k}$ & 1,161 & 1,400 & $3.8 \mathrm{k}$ & $3.1 \mathrm{k}$ & 16 & 16 & 710 & 748 & 521 & 495 \\
\hline pre2 & $245 \mathrm{k}$ & $261 \mathrm{k}$ & 1143 & 1,332 & $6.5 \mathrm{k}$ & $3.8 \mathrm{k}$ & 33 & 26 & 787 & 646 & 100 & 122 \\
\hline kkt_power & $662 \mathrm{k}$ & $684 \mathrm{k}$ & 2,930 & 3459 & $7.2 \mathrm{k}$ & $4.2 \mathrm{k}$ & 50 & 29 & 2,072 & 1,554 & 197 & 262 \\
\hline bcsstk31 & $62 \mathrm{k}$ & $76 \mathrm{k}$ & 223 & 297 & $4.3 \mathrm{k}$ & $3.2 \mathrm{k}$ & 19 & 17 & 283 & 253 & 45 & 50 \\
\hline engin & $117 \mathrm{k}$ & $144 \mathrm{k}$ & 477 & 671 & $4.6 \mathrm{k}$ & $3.1 \mathrm{k}$ & 32 & 27 & 525 & 496 & 96 & 102 \\
\hline shipsec8 & $139 \mathrm{k}$ & $159 \mathrm{k}$ & 471 & 606 & $4.2 \mathrm{k}$ & $3.2 \mathrm{k}$ & 16 & 15 & 353 & 337 & 170 & 178 \\
\hline Transport & $582 \mathrm{k}$ & $645 \mathrm{k}$ & 1487 & 1700 & $5.3 \mathrm{k}$ & $3.2 \mathrm{k}$ & 16 & 10 & 782 & 1230 & 313 & 199 \\
\hline $\mathrm{CO}$ & $1,044 \mathrm{k}$ & $1,070 \mathrm{k}$ & 2,710 & 2,792 & $13.4 \mathrm{k}$ & 10.1 & 45 & 35 & 906 & 783 & 85 & 99 \\
\hline $598 a$ & $104 \mathrm{k}$ & $131 \mathrm{k}$ & 341 & 529 & $5.6 \mathrm{k}$ & $3.6 \mathrm{k}$ & 29 & 24 & 435 & 382 & 103 & 117 \\
\hline $\mathrm{m} 14 \mathrm{~b}$ & $158 \mathrm{k}$ & 190k & 596 & 804 & $5.3 \mathrm{k}$ & $3.5 \mathrm{k}$ & 38 & 31 & 630 & 547 & 123 & 142 \\
\hline roadNet-CA & $35 \mathrm{k}$ & $67 \mathrm{k}$ & 138 & 496 & $3.1 \mathrm{k}$ & $1.3 \mathrm{k}$ & 15 & 8 & 848 & 753 & 178 & 200 \\
\hline great-britain_osm & $26 \mathrm{k}$ & $59 \mathrm{k}$ & 168 & 640 & $4.1 \mathrm{k}$ & $1.4 \mathrm{k}$ & 29 & 11 & 1,202 & 1,151 & 509 & 532 \\
\hline germany_osm & $33 \mathrm{k}$ & $74 \mathrm{k}$ & 242 & 772 & $4.2 \mathrm{k}$ & 1.5 & 31 & 13 & 1,759 & 1,719 & 603 & 617 \\
\hline debr & $505 \mathrm{k}$ & $899 k$ & 1,268 & 2,793 & $68.3 \mathrm{k}$ & $16.9 \mathrm{k}$ & 185 & 74 & 6047 & 1,747 & 11 & 37 \\
\hline
\end{tabular}

Running time is in microseconds

this value are generally higher than those of other $m n c$ values. Observe that for a specific $K$ value, increasing the $m n c$ leads to a decrease in the total message count and an increase in the total volume.

The performance improvement of HP-L over HP increases in terms of parallel SpMV time with increasing $K$ for almost all $m n c$ values. For example, for $m n c=100$, HP$\mathrm{L}$ only achieves a 5 percent improvement in running time over HP at $K=128$, whereas at $K=2048$ this improvement becomes 29 percent. The effect of reducing total message count becomes more apparent in parallel running time with increasing $K$ since the latency component gets more important at high $K$ values.

When we compare HP and HP-L in terms of partitioning times in Table 2, we see that HP-L has higher partitioning overhead as expected. HP-L incurs 7-28 percent, 8-33 percent, 9-41 percent and 15-48 percent slower partitionings for $m n c$ values of 10, 50, 100 and 200, respectively. Although the formation of message nets is not expensive $\left(O\left(p \log _{2} K\right)\right)$, note that the partitioning with HP-L includes the message nets in addition to volume nets, which leads to increased bipartitioning times compared to HP. As $K$ increases, HP-L's partitioning time also increases compared to HP since the number of message nets increases as well.

In Table 3, we present the detailed communication statistics and the parallel SpMV running times and speedups of 30 matrices for $K=512$ and $m n c=50$. In this table, the actual results of HP and HP-L are presented. The unit of the total volume is one kilo-item whereas the unit of the total number of messages is one kilo-message. The columns under "running time" denote the parallel SpMV running time in microseconds and the columns under "speedup" denote the speedups.

In 27 out of 30 matrices, HP-L obtains better speedup values than HP. As also observed and discussed for Table 2, reducing both the total volume and total message count significantly improves the parallel performance. For example, for memchip, HP-L increases the speedup by 71 percent (from 188 to 322 ) by reducing the total message count by 45 percent (from $3.9 \mathrm{k}$ to $2.2 \mathrm{k}$ ). On the other hand, for gsm_106857, having a reduction of 18 percent in the total message count (from $3.8 \mathrm{k}$ to $3.1 \mathrm{k}$ ) by HP-L does not lead to an improved parallel running time.

The reduction in the total number of messages leads to a reduction in the maximum number messages, as observed in Tables 2 and 3. In a similar manner, the increase in the total volume leads to an increase in the maximum volume. The models that provide an upper bound on the maximum message count usually have two communication phases, in each of which the maximum message count is $\sqrt{K}-1$. Compared to these models, apart from the scale-free matrices, although our model does not provide such an upper bound, it usually obtains values below this bound, which is approximately $2(\sqrt{512}-1) \approx 43$ for $K=512$. 
HP-L-10 日 HP-L-50 Ø HP-L-100 $\cong$ HP-L-200 -
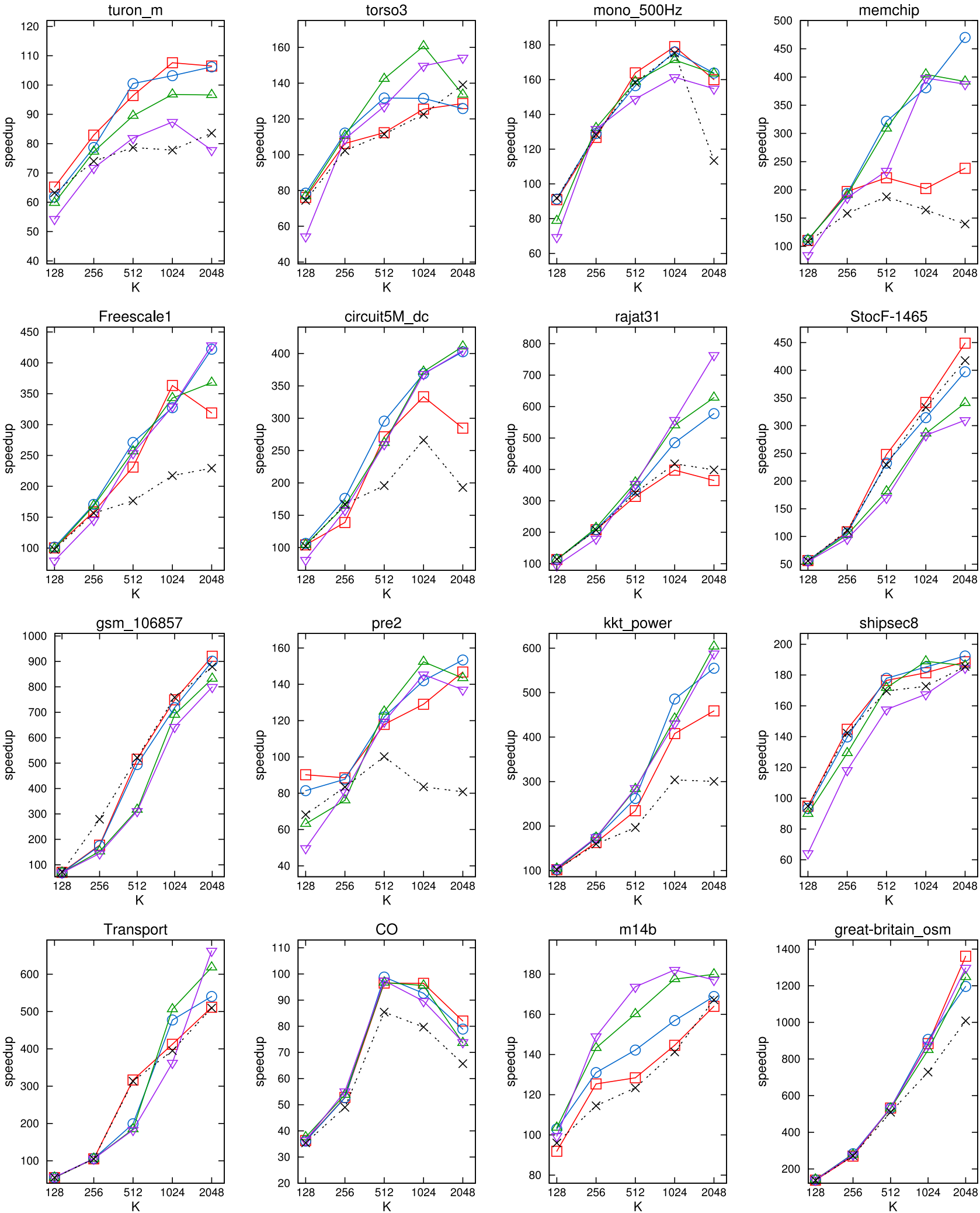

HP-L-10 E HP-L-50 - HP-L-100 HP-L-200 -

Fig. 6. Speedup values for 16 matrices.

As seen in Table 3, a significant reduction in the total message count generally leads to a better performance. There are a couple of basic factors that can be argued to determine whether improving latency cost at the expense of bandwidth cost will result in a better parallel performance. For a partitioning instance, in general, if the average 
message size is relatively high and/or the maximum message count is relatively low, then it can be said that the bandwidth component dominates the latency component. For example, for gsm_106857, the average message size is high and the maximum message count is low compared to the other matrices. Hence, reducing the total message count by 18 percent does not pay off as the latency component is not worth exploiting.

Fig. 6 displays the speevalues of 10,50, 100 and 200. For certain matrices, HP-L drastically changes the scalability by scaling up the parallel SpMV while HP scales down. This is observed for the matrices in the circuit simulation category, and the matrices pre2 and kkt_power. For these matrices, reducing the latency overhead seems to be more important than reducing the bandwidth overhead. HP already exhibits good scalability for the matrices such as m14b, greatbritain_osm and Transport. Reducing latency cost for these matrices pays off as HP-L further improves their scalability. HP and HP-L attain comparable scalability for gsm_106857, StocF-1465 and shipsec8. The latency costs for these matrices are a minor component of their overall communication cost. Among the HP-L schemes, the scalability of HP-L-10 resembles that of $\mathrm{HP}$ the most since HP-L-10 attributes less importance to reducing the latency overhead compared to the other HP-L schemes. For CO and mono_500Hz, both HP and HP-L scale down after a certain number of processors. Nonetheless, HP-L still improves the parallel SpMV running time.

The HP-L schemes with the four different $m n c$ values generally exhibit different parallel performance, as seen in Fig. 6. For any $K$ value, increasing the $m n c$ further decreases the message count and further increases the total volume (see Table 2). How this affects the parallel SpMV running time depends on the communication requirements of the respective partitioning instance. It may pay off to use a high $m n c$ value to further reduce the latency overhead (as is the case for rajat31) or doing so may worsen the parallel running time (as is the case for StocF-1465).

\section{Conclusion}

We have proposed a hypergraph partitioning model in order to parallelize certain types of applications with the objective of reducing the total volume and the total number of messages simultaneously. Our model exploits the recursive bipartitioning paradigm and hence provides the flexibility of using any available hypergraph partitioner. The proposed approach provides a better way for capturing the communication requirements of the target parallel applications. The experimental results showed that the better representation of the communication costs in the proposed hypergraph partitioning model led to a reduction of up to 29 percent in the parallel running time on the average.

As future work, we plan to develop heuristics to dynamically find the best message net cost for each bipartitioning by evaluating the relative importance of the components in the communication cost. We also wish to incorporate the other latency-based communication metrics such as the maximum number of messages communicated by a processor into our model.

\section{ACKNOWLEDGMENTS}

This work is partially supported by the Scientific and Technological Research Council of Turkey (TUBITAK) under project EEEAG-114E545. This article is based upon work from COST Action CA 15109 (COSTNET). We acknowledge PRACE for awarding us access to resources Juqueen (Blue Gene/Q) based in Germany at Jülich Supercomputing Centre.

\section{References}

[1] U. V. Çatalyürek and C. Aykanat, "Hypergraph-partitioningbased decomposition for parallel sparse-matrix vector multiplication," IEEE Trans. Parallel Distrib. Syst., vol. 10, no. 7, pp. 673693, Jul. 1999.

[2] B. Hendrickson, "Graph partitioning and parallel solvers: Has the emperor no clothes? (extended abstract)," in Proc. 5th Int. Symp. Solving Irregularly Struct. Probl. Parallel, 1998, pp. 218-225. [Online]. Available: http://dl.acm.org/citation.cfm?id=646012.677019

[3] B. Hendrickson and T. G. Kolda, "Graph partitioning models for parallel computing," Parallel Comput., vol. 26, no. 12, pp. 15191534, Nov. 2000. [Online]. Available: http://dx.doi.org/10.1016/ S0167-8191(00)00048-X

[4] B. Hendrickson and T. G. Kolda, "Partitioning rectangular and structurally unsymmetric sparse matrices for parallel processing," SIAM J. Sci. Comput., vol. 21, no. 6, pp. 2048-2072, Dec. 1999. [Online]. Available: http://dx.doi.org/10.1137/S1064827598341475

[5] G. Karypis and V. Kumar, "A fast and high quality multilevel scheme for partitioning irregular graphs," SIAM J. Sci. Comput., vol. 20, no. 1, pp. 359-392, Dec. 1998. [Online]. Available: http:// dx.doi.org/10.1137/S1064827595287997

[6] K. Schloegel, G. Karypis, and V. Kumar, "Parallel multilevel algorithms for multi-constraint graph partitioning," in Proc. 6th Int. Euro-ParConf. Parallel Process., vol. 1900, 2000, pp. 296-310. [Online]. Available: http:/ / dx.doi.org/10.1007/3-540-44520-X_39

[7] B. Vastenhouw and R. H. Bisseling, "A two-dimensional data distribution method for parallel sparse matrix-vector multiplication," SIAM Rev., vol. 47, no. 1 pp. 67-95, Jan. 2005. [Online]. Available: http:/ / portal.acm.org/ citation.cfm?id=1055334.1055397

[8] U. Çatalyürek and C. Aykanat, "A hypergraph-partitioning approach for coarse-grain decomposition," in Proc. ACM/IEEE Conf. Supercomputing, 2001, pp. 28-28. [Online]. Available: http:/ / doi.acm.org/10.1145/582034.582062

[9] B. Uçar and C. Aykanat, "Revisiting hypergraph models for sparse matrix partitioning," SIAM Rev., vol. 49, pp. 595-603, Nov. 2007. [Online]. Available: http://portal.acm.org/citation. cfm?id=1330215.1330219

[10] D. Pelt and R. Bisseling, "A medium-grain method for fast 2D bipartitioning of sparse matrices," in Proc. IEEE 28th Int. Parallel Distrib. Process. Symp., May 2014, pp. 529-539.

[11] E. Kayaaslan, B. Ucar, and C. Aykanat, "Semi-two-dimensional partitioning for parallel sparse matrix-vector multiplication," in Proc. IEEE Int. Parallel Distrib. Process. Symp. Workshop, May 2015, pp. 1125-1134.

[12] B. Uçar and C. Aykanat, "Encapsulating multiple communicationcost metrics in partitioning sparse rectangular matrices for parallel matrix-vector multiplies," SIAM J. Sci. Comput., vol. 25, no. 6, pp. 1837-1859, 2004.

[13] M. Deveci, K. Kaya, B. Uçar, and Ümit Çatalyürek, "Hypergraph partitioning for multiple communication cost metrics: Model and methods," J. Parallel Distrib. Comput., vol. 77, pp. 69-83, 2015. [Online]. Available: http://www.sciencedirect.com/science/ article/pii/S0743731514002275

[14] O. Fortmeier, H. Backer, B. F. Auer, and R. Bisseling, "A new metric enabling an exact hypergraph model for the communication volume in distributed-memory parallel applications," Parallel Comput., vol. 39, no. 8, pp. 319-335, 2013. [Online]. Available: http://www.sciencedirect.com/science/article/pii/ S0167819113000690

[15] B. Hendrickson, R. Leland, and S. Plimpton, "An efficient parallel algorithm for matrix-vector multiplication," Int. J. High Speed Comput., vol. 7, pp. 73-88, 1995

[16] J. Lewis, D. Payne, and R. van de Geijn, "Matrix-vector multiplication and conjugate gradient algorithms on distributed memory computers," in Proc. Scalable High-Perform. Comput. Conf., May 1994, pp. 542-550. 
[17] J. G. Lewis and R. A. van de Geijn, “Distributed memory matrixvector multiplication and conjugate gradient algorithms," in Proc. ACM/IEEE Conf. Supercomputing, 1993, pp. 484-492. [Online]. Available: http:/ /doi.acm.org/10.1145/169627.169788

[18] A. Ogielski and W. Aiello, "Sparse matrix computations on parallel processor arrays," SIAM J. Sci. Comput., vol. 14, no. 3, pp. 519530, 1993. [Online]. Available: http://dx.doi.org/10.1137/0914033

[19] A. Buluç and J. Gilbert, "Challenges and advances in parallel sparse matrix-matrix multiplication," in Proc. 37th Int. Conf. Parallel Process, Sep. 2008, pp. 503-510.

[20] A. Buluç and K. Madduri, "Parallel breadth-first search on distributed memory systems," in Proc. Int. Conf. High Perform. Comput. Netw. Storage Anal., 2011, pp. 65:1-65:12. [Online]. Available: http://doi.acm.org/10.1145/2063384.2063471

[21] A. Yoo, A. H. Baker, R. Pearce, and V. E. Henson, "A scalable eigensolver for large scale-free graphs using 2D graph partitioning," in Proc. Int. Conf. High Perform. Comput. Netw. Storage Anal., 2011, pp. 63:1-63:11. [Online]. Available: http://doi.acm. org/10.1145/2063384.2063469

[22] U. V. Çatalyürek, C. Aykanat, and B. Uçar, "On two-dimensional sparse matrix partitioning: Models, methods, and a recipe," SIAM J. Sci. Comput., vol. 32, no. 2, pp. 656-683, Feb. 2010. [Online]. Available: http://dx.doi.org/10.1137/080737770

[23] E. G. Boman, K. D. Devine, and S. Rajamanickam, "Scalable matrix computations on large scale-free graphs using 2D graph partitioning," in Proc. Int. Conf. High Perform. Comput. Netw. Storage Anal., 2013, pp. 50:1-50:12. [Online]. Available: http://doi.acm. org $/ 10.1145 / 2503210.2503293$

[24] R. O. Selvitopi, M. Ozdal, and C. Aykanat, "A novel method for scaling iterative solvers: Avoiding latency overhead of parallel sparse-matrix vector multiplies," IEEE Trans. Parallel Distrib, Syst., vol. 26, no. 3, pp. 632-645, Mar. 2015.

[25] O. Selvitopi and C. Aykanat, "Reducing latency cost in 2D sparse matrix partitioning models," Parallel Comput., vol. 57, pp. 1-24, Sep. 2016. [Online]. Available: http://www.sciencedirect.com/ science/article/pii/S0167819116300138

[26] G. Karypis, R. Aggarwal, V. Kumar, and S. Shekhar, "Multilevel hypergraph partitioning: Applications in VLSI domain," IEEE Trans. Very Large Scale Integr. Syst., vol. 7, no. 1. pp. 69-79, Mar. 1999. [Online]. Available: http://dx.doi.org/10.1109/ 92.748202

[27] F. Pellegrini and J. Roman, "Scotch: A software package for static mapping by dual recursive bipartitioning of process and architecture graphs," in Proc. Int. Conf. Exhibition High-Perform. Comput. Netw. vol. 1067, 1996, pp. 493-498. [Online]. Available: http://dx. doi.org/10.1007/3-540-61142-8_588

[28] M. Wang, S. Lim, J. Cong, and M. Sarrafzadeh, "Multi-way partitioning using bi-partition heuristics," in Proc. Asia South Pacific Des. Autom. Conf., 2000, pp. 1479-1487. [Online]. Available: http:/ / doi.acm.org/10.1145/368434.368865

[29] D. A. Padua, Ed., Encyclopedia of Parallel Computing. New York, NY, USA: Springer, 2011. [Online]. Available: http://dx.doi.org/ 10.1007/978-0-387-09766-4

[30] T. Lengauer, Combinatorial Algorithms for Integrated Circuit Layout. New York, NY, USA: Wiley, 1990.

[31] T. A. Davis and Y. Hu, "The University of Florida sparse matrix collection," ACM Trans. Math. Softw., vol. 38, no. 1, pp. 1:1-1:25, Dec. 2011. [Online]. Available: http://doi.acm.org/10.1145/ 2049662.2049663

[32] S. Balay, et al., "PETSc users manual," Argonne National Laboratory, Lemont, IL, USA, Tech. Rep. ANL-95/11 - Revision 3.5, 2014. [Online]. Available: http://www.mcs.anl.gov/petsc

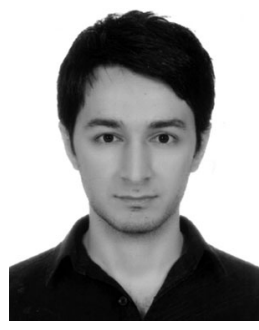

Oguz Selvitopi received the BS degree in computer engineering from Marmara University in 2008 and the MS degree in computer engineering from Bilkent University, Turkey in 2010 . He is currently working toward the $\mathrm{PhD}$ degree. His research interests are parallel computing, scientific computing, and parallel and distributed systems.

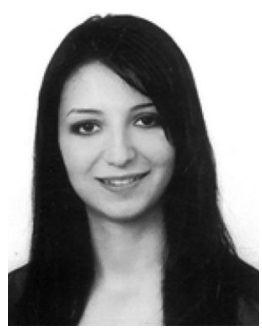

Seher Acer received the BS and MS degrees in computer engineering from Bilkent University, Turkey, from 2009 and 2011, respectively, where she is working toward the $\mathrm{PhD}$ degree. Her research interests are combinatorial scientific computing, graph and hypergraph partitioning, and parallel computing.

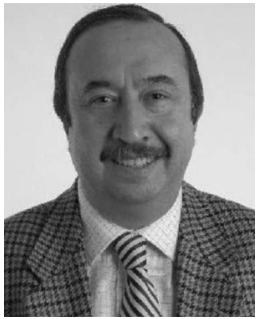

Cevdet Aykanat received the BS and MS degrees from Middle East Technical University, Ankara, Turkey, both in electrical engineering, and the $\mathrm{PhD}$ degree from Ohio State University, Columbus, in electrical and computer engineering. He has served as a member of IFIP Working Group 10.3 (Concurrent System Technology) since 2004 and as an associate editor of IEEE Transactions of Parallel and Distributed Systems between 2008 and 2012. He worked at the Intel Supercomputer Systems Division, Beaverton, Oregon, as a research associate. Since 1989, he has been affiliated with the Department of Computer Engineering, Bilkent University, Ankara, Turkey, where he is currently a professor. His research interests mainly include parallel computing, parallel scientific computing and its combinatorial aspects. He has (co)authored about 90 technical papers published in academic journals indexed in ISI and his publications received about 1,000 citations in ISI indexes. He is the recipient of the 1995 Young Investigator Award of The Scientific and Technological Research Council of Turkey and 2007 Parlar Science Award.

$\triangleright$ For more information on this or any other computing topic, please visit our Digital Library at www.computer.org/publications/dlib. 